\title{
Global Solutions for Abstract Differential Equations with Non-Instantaneous Impulses
}

\author{
Michelle Pierri, Hernán R. Henríquez and Andréa Prokopczyk
}

\begin{abstract}
In this note we study the existence of global solutions for a class of impulsive abstract differential equations with non-instantaneous impulses. Specifically, we establish the existence of mild solutions on $[0, \infty)$ and the existence of $\mathcal{S}$-asymptotically $\omega$-periodic mild solutions. Our results are based on the Hausdorff measure of non-compactness. Some applications involving partial differential equations are considered.
\end{abstract}

Mathematics Subject Classification. 34K30, 34K45, 35R12, 47D06.

Keywords. First-order abstract Cauchy problem, impulsive differential equations, mild solutions, asymptotically periodic solutions, strongly continuous semigroups of linear operators.

\section{Introduction}

In this paper we study the existence of global solutions for a class of abstract differential equations with non-instantaneous impulses of the form

$$
\begin{aligned}
u^{\prime}(t) & =A u(t)+f(t, u(t)), \quad t \in\left[s_{i}, t_{i+1}\right], i \in \mathbb{N}, \\
u(t) & =g_{i}\left(t, N_{i}(t)(u)\right), \quad t \in\left(t_{i}, s_{i}\right], i \in \mathbb{N}, \\
u(0) & =x_{0},
\end{aligned}
$$

where $A: D(A) \subseteq X \rightarrow X$ is the infinitesimal generator of a $C_{0}$-semigroup of bounded linear operators $(T(t))_{t>0}$ defined on a Banach space $(X,\|\cdot\|)$, $x_{0} \in X$ and $0=t_{0}=s_{0}<t_{1}<s_{1}<\cdots<t_{i}<s_{i}<t_{i+1}<\ldots$ are pre-fixed real numbers, $N_{i}(t): C\left(\left[t_{i}, s_{i}\right] ; X\right) \rightarrow X$ are continuous maps for $t \in\left[t_{i}, s_{i}\right]$, the function $t \mapsto N_{i}(t)(u)$ is continuous for each $u \in C\left(\left[t_{i}, s_{i}\right] ; X\right)$, $g_{i} \in C\left(\left[t_{i}, s_{i}\right] \times X ; X\right)$ for all $i \in \mathbb{N}$ and $f:[0, \infty) \times X \rightarrow X$ is a suitable function. Here, as throughout the text, for an interval $I \subseteq[0, \infty)$, we denote by $C(I ; X)$ the space consisting of bounded continuous functions from $I$ into $X$ provided with the norm of uniform convergence.

Hernán R. Henríquez was partially supported by CONICYT, under Grant FONDECYT 1130144, and DICYT-USACH. 
The literature on impulsive abstract differential equations is very extensive and consider basically problems in which the impulses are abrupt and instantaneous. Concerning to the general motivations of the theory, its most relevant developments and the current status of this class of problems, we refer the reader to $[1,2,5,7,9-12,15,21,22,25,26,29,31,34,35,37-43]$ and the references therein. In addition, concerning the existence of global and almost periodic type solutions for differential equations with impulses we cite the papers [3, 15,23,26,38,40-42], the recent book by Stamov [39] and the references therein.

The study of abstract differential equations with non-instantaneous impulses was initiated recently by Hernández and O'Regan in [20]. In the abstract model analyzed in [20], the impulses are triggered abruptly at the instants $t_{i}$ and their action remains during a finite time interval of the form $\left[t_{i}, s_{i}\right]$. As pointed in [20], there are many different motivations for the study of this type of problems. As example, from [20] we note the following simplified situation concerning the hemodynamical equilibrium of a person. In the case of a decompensation (for example, high or low levels of glucose) one can prescribe some intravenous drugs (insulin). As the entry of drugs into the bloodstream and the consequent absorption by the body are gradual and continuous processes, we can interpret this situation as an impulsive action which starts abruptly at a certain instant and stays active on a finite time interval.

In this paper, we continue the development in [20]. Specifically, we discuss the existence of mild solutions on $[0, \infty)$ and the existence of $\mathcal{S}$ asymptotically $\omega$-periodic mild solutions for (1.1)-(1.3). Furthermore, we consider the more realistic situation in which the impulsive action is not instantaneous but depends on its accumulation over the entire time interval in which acts.

We next introduce some additional notations, definitions and results used in this paper. Let $\left(Z,\|\cdot\|_{Z}\right)$ and $\left(W,\|\cdot\|_{W}\right)$ be Banach spaces. In this paper, we denote by $\mathcal{L}(Z, W)$ the Banach space of bounded linear operators from $Z$ into $W$ endowed with the norm of operators denoted by $\|\cdot\|_{\mathcal{L}(Z, W)}$, and we abbreviate this notation to $\mathcal{L}(Z)$ and $\|\cdot\|_{\mathcal{L}(Z)}$ when $Z=W$. In addition, $B_{r}(z, Z)$ denotes the closed ball in $Z$ with center at $z \in Z$ and radius $r$. When the space $Z$ is clear from the context, we write simply $B_{r}(z)$ instead of $B_{r}(z, Z)$. Henceforth, $M \geq 1$ and $\sigma \in \mathbb{R}$ are constants such that $\|T(t)\| \leq M e^{\sigma t}$ for all $t \geq 0$ and $C_{i}=\sup _{t \in\left[s_{i}, t_{i+1}\right]} e^{\sigma\left(t-s_{i}\right)}$, for $i \in \mathbb{N}_{0}$. For additional details on semigroup theory, we refer the reader to [30].

To treat with the impulsive action, we consider the vector space $\mathcal{P C}(X)$ which is formed by all functions $u:[0, \infty) \rightarrow X$ such that $u(\cdot)$ is continuous at $t \neq t_{i}, u\left(t_{i}^{-}\right)=u\left(t_{i}\right)$ and $u\left(t_{i}^{+}\right)$exists for all $i \in \mathbb{N}$. For $u \in \mathcal{P C}(X)$ and $i \in \mathbb{N}_{0}$, we denote by $\tilde{u}_{i}$ the function $\tilde{u}_{i} \in C\left(\left[t_{i}, t_{i+1}\right] ; X\right)$ given by

$$
\widetilde{u}_{i}(t)= \begin{cases}u(t), & \text { for } t \in\left(t_{i}, t_{i+1}\right] \\ u\left(t_{i}^{+}\right), & \text {for } t=t_{i} .\end{cases}
$$


In addition, for $B \subseteq \mathcal{P C}(X), t \geq 0$ and $i \in \mathbb{N}_{0}$, we use the notations $\widetilde{B}_{i}$ and $B(t)$ for the sets $\widetilde{B}_{i}=\left\{\tilde{u}_{i}: u \in B\right\}$ and $B(t)=\{u(t): u \in B\}$.

We denote by $\mathcal{P C}_{b}(X)$ the subspace of $\mathcal{P C}(X)$ consisting of bounded functions endowed with the norm of uniform convergence denoted by $\|\cdot\|_{\mathcal{P C}(X)}$. It is well known that $\mathcal{P C}_{b}(X)$ is a Banach space. Moreover, the following compactness criterion holds.

Lemma 1.1. Let $B \subseteq \mathcal{P C}_{b}(X)$. Assume $\widetilde{B}_{i}$ is relatively compact in $C\left(\left[t_{i}, t_{i+1}\right]\right.$, $X)$ for all $i \in \mathbb{N}_{0}$ and $u(t) \rightarrow 0$ as $t \rightarrow \infty$ uniformly for $u \in B$. Then $B$ is relatively compact in $\mathcal{P C}_{b}(X)$.

From this Lemma we deduce the following Ascoli-Arzelá criterion.

Corollary 1.1. Let $B \subseteq \mathcal{P C}_{b}(X)$. Assume that the following conditions hold.

(a) $B(t)$ is relatively compact in $X$ for all $t \geq 0$ and $B$ is equicontinuous at $t \neq t_{i}$, for all $i \in \mathbb{N}$.

(b) For each $i \in \mathbb{N}, \lim _{t \rightarrow t_{i}^{+}} u(t)$ exists uniformly for $u \in B$ and $\lim _{t \rightarrow \infty}$ $u(t)=0$ uniformly for $u \in B$.

Then $B$ is relatively compact in $\mathcal{P C}_{b}(X)$.

Proof. It is immediate from condition (b) that $\widetilde{B}_{i}$ is an equicontinuous set at $t \in\left[t_{i}, t_{i+1}\right]$. Moreover, it follows from (a) that $\widetilde{B}_{i}(t)$ is relatively compact in $X$ for all $t \in\left(t_{i}, t_{i+1}\right]$. In addition, for each $\varepsilon>0$, it follows from (b) that there exists $\delta>0$ such that $\left\|u\left(t_{i}^{+}\right)-u\left(t_{i}+\delta\right)\right\| \leq \varepsilon$, which implies that $\widetilde{B}_{i}\left(t_{i}\right) \subseteq$ $\widetilde{B}_{i}\left(t_{i}+\delta\right)+B_{\varepsilon}(0)$. This yields that $\widetilde{B}_{i}\left(t_{i}\right)$ is relatively compact. Consequently, $\widetilde{B}_{i}, i \in \mathbb{N}_{0}$, is relatively compact in $C\left(\left[t_{i}, t_{i+1}\right] ; X\right)$. We complete the proof using condition (b) and Lemma 1.1.

For the convenience of the reader, we recall below some properties of the concept of Hausdorff measure of non-compactness. For general information about this topic the reader can see $[4,14]$.

Definition 1.1. Let $B$ be a bounded subset of a metric space $Y$. The Hausdorff measure of non-compactness of $B$ is defined by

$\gamma(B)=\inf \{\varepsilon>0: B$ has a finite cover by closed balls of radius $\varepsilon\}$.

For a bounded set $B \subseteq X$, we next denote by $\overline{c o}(B)$ the closed convex hull of the set $B$. Moreover, if $B$ is a set of functions, $B(t)=\{v(t): v \in B\}$.

Remark 1.1 [4]. Let $B, B_{1}, B_{2} \subseteq X$ be bounded sets. The Hausdorff measure of non-compactness has the following properties.

(a) If $B_{1} \subseteq B_{2}$, then $\gamma\left(B_{1}\right) \leqslant \gamma\left(B_{2}\right)$.

(b) $\gamma(B)=\gamma(\bar{B})=\gamma(\overline{c o}(B))$ and $\gamma(\lambda B)=|\lambda| \gamma(B)$ for all $\lambda \in \mathbb{R}$.

(c) $\gamma(B)=0$ if and only if $B$ is totally bounded.

(d) $\gamma\left(B_{1} \cup B_{2}\right)=\max \left\{\gamma\left(B_{1}\right), \gamma\left(B_{2}\right)\right\}$ and $\gamma\left(B_{1}+B_{2}\right) \leqslant \gamma\left(B_{1}\right)+\gamma\left(B_{2}\right)$.

In what follows, we will use the symbols $\zeta$ and $\gamma$ to denote the Hausdorff measures of non-compactness on $X$ and $C([a, b] ; X)$, respectively.

Lemma 1.2. Let $W \subseteq C([a, b] ; X)$. If $W$ is bounded and equicontinuous, then the set $\overline{c o}(W)$ is also bounded and equicontinuous. 
Lemma 1.3. Let $W \subseteq C([a, b] ; X)$ be a bounded set. Then $\zeta(W(t)) \leqslant \gamma(W)$ for all $t \in[a, b]$. Furthermore, if $W$ is equicontinuous on $[a, b]$, then the function $t \rightarrow \zeta(W(t))$ is continuous on $[a, b]$ and $\gamma(W)=\sup \{\zeta(W(t)): t \in[a, b]\}$.

We note that a set $W \subseteq L^{1}([a, b] ; X)$ is said to be uniformly integrable if there exists a positive function $k \in L^{1}([a, b])$ such that $\|w(t)\| \leq k(t)$ a.e. for all $w \in W$.

Lemma 1.4 [16, Theorem 3.1]. Assume that $X$ is a separable Banach space. If $W \subseteq L^{1}([a, b] ; X)$ is uniformly integrable, then the function $t \rightarrow \zeta(\{W(t)\}$ is measurable and

$$
\zeta\left(\left\{\int_{a}^{b} w(s) d s: w \in W\right\}\right) \leqslant \int_{a}^{b} \zeta(\{w(s): w \in W\}) d s .
$$

The next property has been studied by several authors $[6,44]$ under different hypotheses. We establish it here to unify the presentation and avoid some unnecessary hypotheses.

Lemma 1.5. Let $(Y, d)$ be a metric space and let $D \subseteq Y$ be a bounded set. Then there exists a countable set $D_{0} \subseteq D$ such that $\gamma\left(D_{0}\right)=\gamma(D)$.

Corollary 1.2. Let $W \subseteq L^{1}([a, b] ; X)$ be a uniformly integrable set and $m \in$ $L^{1}([a, b])$ be a positive function such that $\zeta(W(t)) \leq m(t)$ a.e. If $F: L^{1}([a, b]$; $X) \rightarrow X$ is the map given by $F(u)=\int_{a}^{b} u(s) d s$, then $\zeta(F(W)) \leq \int_{a}^{b} m(s) d s$.

Proof. From Lemma 1.5, there exists a countable set $W_{0}=\left\{w_{n}: n \in \mathbb{N}\right\} \subseteq$ $W$ such that $\zeta(F(W))=\zeta\left(F\left(W_{0}\right)\right)$. It follows from [27, Proposition 2.2.6] that there exist $Z_{n} \subseteq[a, b]$ with Lebesgue measure $\lambda\left(Z_{n}\right)=0$ such that $w_{n}\left([a, b] \backslash Z_{n}\right)$ is separable. Redefining $w_{n}$ on a set of measure zero, which does not change the value $F\left(w_{n}\right)$, we can assume that $\cup_{n=1}^{\infty} w_{n}([a, b])$ is separable. Thus, there exists a separable closed subspace $X_{0}$ of $X$ such that $W_{0}([a, b]) \subseteq$ $X_{0}$.

We identify $F$ with its restriction to $L^{1}\left([a, b] ; X_{0}\right)$. Since $W_{0}$ is uniformly integrable, using Lemma 1.4 we obtain that $\zeta\left(F\left(W_{0}\right)\right) \leq \int_{0}^{a} \zeta\left(W_{0}(s)\right) d s$.

Definition 1.2. A continuous map $F: X \rightarrow X$ is said to be a $\gamma$ - $k$-set contraction, $k \in(0,1)$, if for all bounded set $B \subset X, \gamma(F(B)) \leqslant k \gamma(B)$ and $\mathrm{F}$ is said to be $\gamma$-condensing if $\gamma(F(B))<\gamma(B)$ for every bounded subset $B$ of $X$ with $\gamma(B)>0$.

The following result was established by Darbo [8] in 1955 for $\gamma$ - $k$-set contractions, and for Sadovskii [36] in 1967 for $\gamma$-condensing maps.

Theorem 1.1. Assume that $M$ is a nonempty bounded closed and convex subset of a Banach space $X$. Let $F: M \rightarrow M$ be a $\gamma$-condensing map. Then $F$ has a fixed point in $M$.

The following result is a recent extension of Theorem 1.1 established in [24]. 
Theorem 1.2. Let $B$ be a closed and convex subset of a Banach space $Z$ and $F: B \rightarrow B$ be a continuous map such that $F(B)$ is bounded. For each bounded subset $D \subseteq B$, denote $F^{1}(D)=F(D)$ and $F^{n}(D)=F\left(\overline{c o}\left(F^{n-1}(D)\right)\right), n \in$ $2,3, \ldots$ If there exist $n_{0} \in \mathbb{N}$ and $r \in[0,1)$ such that $\gamma\left(F^{n_{0}}(D)\right) \leqslant r \gamma(D)$, for all bounded set $D \subseteq B$, then $F$ has a fixed point.

This paper has three sections. In Sect. 2 we study the existence of global solutions for (1.1)-(1.3). In Sect. 3, some applications involving the heat equation are considered.

\section{Existence of Global Solutions}

In this section we discuss the existence of global mild solutions for the problem (1.1)-(1.3). In the remainder of this work, for $u \in \mathcal{P C}(X)$ and $i \in \mathbb{N}$, we use the notation $v_{i}$ for the function

$$
v_{i}(t)= \begin{cases}u(t), & t \in\left(t_{i}, s_{i}\right], \\ u\left(t_{i}^{+}\right), & t=t_{i} .\end{cases}
$$

Following [20] we adopt the following concept of solution.

Definition 2.1. A function $u \in \mathcal{P C}(X)$ is called a mild solution of problem (1.1)-(1.3) if $u(0)=x_{0}, u(t)=g_{j}\left(t, N_{j}(t)\left(v_{j}\right)\right)$ for all $t \in\left(t_{j}, s_{j}\right]$ and each $j \in \mathbb{N}$, and

$$
\begin{aligned}
& u(t)=T(t) x_{0}+\int_{0}^{t} T(t-\tau) f(\tau, u(\tau)) d \tau, \quad t \in\left[0, t_{1}\right], \\
& u(t)=T\left(t-s_{i}\right) u\left(s_{i}\right)+\int_{s_{i}}^{t} T(t-\tau) f(\tau, u(\tau)) d \tau, \quad t \in\left[s_{i}, t_{i+1}\right], i \in \mathbb{N} .
\end{aligned}
$$

To establish our results we introduce a number of conditions on $f, g_{i}$, $N_{i}$. In what follows we denote $J=\cup_{i=0}^{\infty}\left[s_{i}, t_{i+1}\right]$ and $J^{\prime}=\cup_{i=1}^{\infty}\left[t_{i}, s_{i}\right]$.

$\left(\mathbf{H}_{\mathbf{1}}\right)$ There are positive constants $L_{g_{i}}$ such that $\left\|g_{i}(t, x)-g_{i}(t, y)\right\| \leq L_{g_{i}} \|$ $x-y \|$ for all $x, y \in X, t \in\left[t_{i}, s_{i}\right]$ and each $i \in \mathbb{N}$.

$\left(\mathbf{H}_{2}\right)$ The map $f:[0, \infty) \times X \rightarrow X$ satisfies the Carathéodory conditions. That is, $f(\cdot, x)$ is measurable for all $x \in X$ and $f(t, \cdot)$ is continuous for almost all $t \in J$.

$\left(\mathbf{H}_{3}\right)$ There are functions $m_{f}, h_{f} \in L_{l o c}^{1}\left(J ; \mathbb{R}^{+}\right)$and $\Phi_{i} \in C\left([0, \infty) ; \mathbb{R}^{+}\right)$, $i \in \mathbb{N}_{0}$, non-decreasing such that

$$
\|f(t, x)\| \leq m_{f}(t) \Phi_{i}(\|x\|)+h_{f}(t)
$$

for all $x \in X$ and almost all $t \in\left[s_{i}, t_{i+1}\right]$.

$\left(\mathbf{H}_{4}\right)$ There exists a function $H \in L_{l o c}^{1}\left(J ; \mathbb{R}^{+}\right)$such that

$$
\zeta(f(t, B)) \leqslant H(t) \zeta(B)
$$

for almost all $t \in J$ and every bounded set $B \subseteq X$.

$\left(\mathbf{H}_{\mathbf{5}}\right)$ There exist constants $\mu_{i}>0, i \in \mathbb{N}$ such that

$$
\gamma\left(\left\{N_{i}(\cdot)(v): v \in W_{i}\right\}\right) \leqslant \mu_{i} \gamma\left(W_{i}\right)
$$

for every bounded set $W_{i} \subseteq C\left(\left[t_{i}, s_{i}\right] ; X\right)$. 
When condition $\left(\mathbf{H}_{\mathbf{5}}\right)$ holds, the maps $N_{i}(\cdot): C\left(\left[t_{i}, s_{i}\right]\right.$; $X) \rightarrow C\left(\left[t_{i}, s_{i}\right] ; X\right), i \in \mathbb{N}$, given by $\left(N_{i}(\cdot) u\right)(t)=N_{i}(t) u$ are uniformly bounded on bounded sets. In this case, we use the notation

$$
\nu_{i, R}=\sup \left\{\left\|N_{i}(t)(v)\right\|: t \in\left[t_{i}, s_{i}\right], v \in C\left(\left[t_{i}, s_{i}\right] ; X\right),\|v\|_{\infty} \leq R\right\} .
$$

Remark 2.1. If condition $\left(\mathbf{H}_{\mathbf{5}}\right)$ holds, then the maps $\widetilde{N}_{i}(\cdot): C\left(\left[t_{i}, s_{i}\right] ; X\right) \rightarrow$ $C\left(\left[t_{i}, s_{i}\right] ; X\right), i \in \mathbb{N}$, defined by $\widetilde{N}_{i}(v)(t)=N_{i}(t)(v)$, are continuous. In fact, if $\left(v_{n}\right)_{n}$ is a sequence convergent to $v$ in $C\left(\left[t_{i}, s_{i}\right] ; X\right)$, then the set $W=\left\{v_{n}\right.$ : $n \in \mathbb{N}\}$ is relatively compact in $C\left(\left[t_{i}, s_{i}\right] ; X\right)$, which implies that $\left\{N_{i}(\cdot)\left(v_{n}\right)\right.$ : $n \in \mathbb{N}\}$ is also relatively compact. Therefore, there exists a subsequence $\left(v_{n_{k}}\right)_{k}$ of $\left(v_{n}\right)_{n}$ such that $N_{i}(\cdot)\left(v_{n_{k}}\right) \rightarrow N_{i}(\cdot)(v)$ as $k \rightarrow \infty$ in $C\left(\left[t_{i}, s_{i}\right] ; X\right)$. Since this property is independent of the sequence $\left(v_{n_{k}}\right)_{k}$, we obtain that $N_{i}(\cdot)\left(v_{n}\right) \rightarrow N_{i}(\cdot)(v)$ as $n \rightarrow \infty$.

In order to show the generality of our presentation, we exhibit below a pair of simple examples of families $\left(N_{i}\right)_{i \in \mathbb{N}}$ that verify the condition $\mathbf{H}_{\mathbf{5}}$.

Example 2.1. Let $Q_{i}:\left[t_{i}, s_{i}\right] \rightarrow \mathcal{L}(X), i \in \mathbb{N}$, be a strongly continuous operator map and let

$$
N_{i}(t)(v)=Q_{i}(t) v(t), v \in C\left(\left[t_{i}, s_{i}\right] ; X\right), t \in\left[t_{i}, s_{i}\right], i \in \mathbb{N} .
$$

Since $\left\{Q_{i}(t): t \in\left[t_{i}, s_{i}\right]\right\}$ is bounded for the norm of operators, then $N_{i}(\cdot)(v)$ is continuous on $\left[t_{i}, s_{i}\right]$ for each $v \in C\left(\left[t_{i}, s_{i}\right] ; X\right)$ and $N_{i}(\cdot)$ is Lipschitz continuous. In particular, this occurs for $Q(t)=I$. In this case, Eq. (1.2) is reduced to $u(t)=g_{i}(t, u(t))$.

Example 2.2. Let $k_{i}:\left[t_{i}, s_{i}\right] \times\left[t_{i}, s_{i}\right] \times X \rightarrow X, i \in \mathbb{N}$, be a continuous function. Assume that $k_{i}$ takes bounded sets into bounded sets and there are positive functions $\mu_{i} \in L^{1}\left(\left[t_{i}, s_{i}\right]\right)$ such that $\zeta\left(\left\{k_{i}(\tau, t, x): x \in B\right\}\right) \leq$ $\mu_{i}(\tau) \zeta(B)$, for every bounded set $B \subseteq X$. Then the maps

$$
N_{i}(t)(v)=\int_{t_{i}}^{s_{i}} k_{i}(\tau, t, v(\tau)) d \tau, v \in C\left(\left[t_{i}, s_{i}\right] ; X\right), t \in\left[t_{i}, s_{i}\right],
$$

satisfy condition $\left(\mathbf{H}_{\mathbf{5}}\right)$. In fact, it is clear that $N_{i}(\cdot)(v)$ is continuous for each $v \in C\left(\left[t_{i}, s_{i}\right] ; X\right)$. Moreover, applying Corollary 1.2 , we have

$$
\zeta\left(N_{i}(t)(W)\right) \leq \int_{t_{i}}^{s_{i}} \mu_{i}(\tau) d \tau \gamma(W)
$$

for all bounded set $W \subseteq C\left(\left[t_{i}, s_{i}\right] ; X\right)$. We note that in this case, Eq. (1.2) is reduced to $u(t)=g_{i}\left(t, \int_{t_{i}}^{s_{i}} k_{i}(\tau, t, u(\tau)) d \tau\right)$.

We can establish now our first results on the existence of global solutions.

Theorem 2.1. Assume that conditions $\left(\mathbf{H}_{\mathbf{1}}\right)-\left(\mathbf{H}_{\mathbf{5}}\right)$ are fulfilled,

$$
L_{g_{i+1}} \mu_{i+1}<1
$$


for each $i \in \mathbb{N}_{0}$ and there exist constants $R_{1}(i), R_{2}(i+1) \geqslant 0$ such that

$$
\begin{gathered}
M C_{i} R_{2}(i)+M \sup _{s_{i} \leq t \leq t_{i+1}} \int_{s_{i}}^{t} e^{\sigma(t-\tau)}\left(\Phi_{i}\left(R_{1}(i)\right) m_{f}(\tau)+h_{f}(\tau)\right) d \tau \leq R_{1}(i), \\
L_{g_{i+1}} \nu_{i+1, R_{2}(i+1)}+\sup _{t_{i+1} \leq t \leq s_{i+1}}\left\|g_{i+1}(t, 0)\right\| \leq R_{2}(i+1),
\end{gathered}
$$

where $R_{2}(0)=\left\|x_{0}\right\|$. Then the problem (1.1)-(1.3) has at least one mild solution $u \in \mathcal{P C}(X)$.

Proof. We consider the map $G_{i}: C\left(\left[t_{i}, s_{i}\right] ; X\right) \rightarrow C\left(\left[t_{i}, s_{i}\right] ; X\right), i \in \mathbb{N}$, given by

$$
G_{i}(v)(t)=g_{i}\left(t, N_{i}(t)(v)\right), t_{i} \leq t \leq s_{i} .
$$

It follows from our general hypotheses and Remark 2.1 that $G_{i}$ is a continuous map. Moreover, if $v \in C\left(\left[t_{i}, s_{i}\right] ; X\right)$ with $\sup _{t_{i} \leq t \leq s_{i}}\|v(t)\| \leq R_{2}(i)$, then

$$
\begin{aligned}
\left\|G_{i}(v)(t)\right\| & \leq\left\|g_{i}\left(t, N_{i}(t)(v)\right)-g_{i}(t, 0)\right\|+\left\|g_{i}(t, 0)\right\| \\
& \leq L_{g_{i}} \nu_{i, R_{2}(i)}+\sup _{t_{i} \leq t \leq s_{i}}\left\|g_{i}(t, 0)\right\|,
\end{aligned}
$$

which implies that $G_{i}\left(B_{R_{2}(i)}(0)\right) \subseteq B_{R_{2}(i)}(0)$. Moreover, if $W \subset C\left(\left[t_{i}, s_{i}\right] ; X\right)$ is bounded with $\gamma(W)>0$, and $W^{\prime}=\left\{N_{i}(\cdot)(v): v \in W\right\}$, from $\left(\left(\mathbf{H}_{\mathbf{1}}\right)\right)$ and $\left(\mathbf{H}_{5}\right)$, we obtain

$$
\gamma\left(G_{i}(W)\right) \leq L_{g_{i}} \gamma\left(W^{\prime}\right) \leq L_{g_{i}} \mu_{i} \gamma(W)<\gamma(W) .
$$

Consequently, $G_{i}$ is a condensing map, and using Theorem 1.1 we infer that there exists a fixed point $v_{i}$ of $G_{i}$.

We define $\Gamma_{i}: C\left(\left[s_{i}, t_{i+1}\right] ; X\right) \rightarrow C\left(\left[s_{i}, t_{i+1}\right] ; X\right), i \in \mathbb{N}_{0}$, by

$$
\begin{aligned}
\left(\Gamma_{i} v\right)(t) & =T\left(t-s_{i}\right)\left(v_{i}\left(s_{i}\right)\right)+\int_{s_{i}}^{t} T(t-\tau) f(\tau, v(\tau)) d \tau, t \in\left[s_{i}, t_{i+1}\right], \\
v & \in C\left(\left[s_{i}, t_{i+1}\right] ; X\right),
\end{aligned}
$$

where $v_{0}\left(s_{0}\right)=x_{0}$. Since the function $\tau \mapsto f(\tau, v(\tau))$ is integrable on $\left[s_{i}, t_{i+1}\right]$, we infer that $\Gamma_{i}$ is well defined. Moreover, combining $\left(\mathbf{H}_{\mathbf{2}}\right),\left(\mathbf{H}_{\mathbf{3}}\right)$ and the Lebesgue dominated convergence theorem we deduce that $\Gamma_{i}$ is a continuous map. that

On the other hand, if $\sup _{s_{i} \leq t \leq t_{i+1}}\|v(t)\| \leq R_{1}(i)$, it follows from (2.5)

$$
\begin{aligned}
\left\|\left(\Gamma_{i} v\right)(t)\right\| \leqslant & \left\|T\left(t-s_{i}\right)\left(v_{i}\left(s_{i}\right)\right)\right\|+\left\|\int_{s_{i}}^{t} T(t-\tau) f(\tau, v(\tau)) d \tau\right\| \\
\leqslant & M C_{i} R_{2}(i)+M \Phi_{i}\left(R_{1}(i)\right) \int_{s_{i}}^{t} e^{\sigma(t-\tau)} m_{f}(\tau) d \tau \\
& +M \int_{s_{i}}^{t} e^{\sigma(t-\tau)} h_{f}(\tau) d \tau \\
\leqslant & R_{1}(i)
\end{aligned}
$$

which implies that $\Gamma_{i}\left(B_{R_{1}(i)}(0)\right) \subseteq B_{R_{1}(i)}(0)$. 
Let $W$ be a bounded subset of $B_{R_{1}(i)}(0), t \in\left[s_{i}, t_{i+1}\right]$ and $\tau \in\left[s_{i}, t\right]$. It follows directly from the Definition 1.1 that

$$
\begin{aligned}
\zeta(\{T(t-\tau) f(\tau, v(\tau)): v \in W\}) & \leq M e^{\sigma(t-\tau)} \zeta(\{f(\tau, v(\tau)): v \in W\}) \\
& \leq M e^{\sigma(t-\tau)} H(\tau) \gamma(W) .
\end{aligned}
$$

Using now Corollary 1.2, we obtain

$$
\zeta\left(\Gamma_{i}(W)(t)\right) \leq M \int_{s_{i}}^{t} e^{\sigma(t-\tau)} H(\tau) d \tau \gamma(W) \leq M C_{i} \int_{s_{i}}^{t} H(\tau) d \tau \gamma(W) .
$$

In addition, a simple estimate applying condition $\left(\mathbf{H}_{\mathbf{3}}\right)$ shows that $\Gamma_{i}(W)$ is an equicontinuous subset of $C\left(\left[s_{i}, t_{i+1}\right] ; X\right)$. Therefore, making use of Lemma 1.3, we can write

$$
\gamma\left(\Gamma_{i}(W)\right) \leq M C_{i} \int_{s_{i}}^{t_{i+1}} H(\tau) d \tau \gamma(W) .
$$

We now evaluate $\gamma\left(\Gamma_{i}^{2}(W)\right)=\gamma\left(\Gamma_{i}\left(W^{\prime}\right)\right)$, where $W^{\prime}=\overline{c o}\left(\Gamma_{i}(W)\right)$. It follows from Remark 1.1, condition $\left(\mathbf{H}_{\mathbf{4}}\right)$ and (2.6) that

$$
\begin{aligned}
\zeta\left(\left\{f(\tau, v(\tau)): v \in W^{\prime}\right\}\right) & \leq H(\tau) \zeta\left(W^{\prime}(\tau)\right) \\
& =H(\tau) \zeta\left(\Gamma_{i}(W)(\tau)\right) \\
& \leq H(\tau) M C_{i} \int_{s_{i}}^{\tau} H(\xi) d \xi \gamma(W) .
\end{aligned}
$$

Hence, repeating our previous arguments, we can write

$$
\begin{aligned}
\gamma\left(\Gamma_{i}^{2}(W)(t)\right) & \leq M \int_{s_{i}}^{t} e^{\sigma(t-\tau)} H(\tau) M C_{i} \int_{s_{i}}^{\tau} H(\xi) d \xi d \tau \gamma(W) \\
& \leq M^{2} C_{i}^{2} \int_{s_{i}}^{t} \int_{s_{i}}^{\tau} H(\tau) H(\xi) d \xi d \tau \gamma(W) \\
& =\frac{1}{2} M^{2} C_{i}^{2}\left(\int_{s_{i}}^{t} H(\tau) d \tau\right)^{2} \gamma(W)
\end{aligned}
$$

and

$$
\gamma\left(\Gamma_{i}^{2}(W)\right) \leq \frac{1}{2} M^{2} C_{i}^{2}\left(\int_{s_{i}}^{t_{i+1}} H(\tau) d \tau\right)^{2} \gamma(W) .
$$

Proceeding inductively, we obtain

$$
\gamma\left(\Gamma_{i}^{n}(W)\right) \leq \frac{1}{n !} M^{n} C_{i}^{n}\left(\int_{s_{i}}^{t_{i+1}} H(\tau) d \tau\right)^{n} \gamma(W) .
$$

Since $\frac{1}{n !} M^{n} C_{i}^{n}\left(\int_{s_{i}}^{t_{i+1}} H(\tau) d \tau\right)^{n} \rightarrow 0$ as $n \rightarrow \infty$, applying Theorem 1.2 we infer that there exists $u_{i} \in C\left(\left[s_{i}, t_{i+1}\right] ; X\right)$ such that $\Gamma_{i}\left(u_{i}\right)=u_{i}$.

Using this inductive construction, we are led to define

$$
u(t)=\left\{\begin{array}{l}
u_{i}(t), \quad t \in\left[s_{i}, t_{i+1}\right), \quad i \in \mathbb{N}_{0}, \\
v_{i}(t), \quad t \in\left[t_{i}, s_{i}\right], \quad i \in \mathbb{N}
\end{array}\right.
$$

It is not difficult to see that $u \in \mathcal{P C}(X)$ is a mild solution of problem (1.1)$(1.3)$. 
Corollary 2.1. Assume that conditions $\left(\mathbf{H}_{\mathbf{1}}\right)-\left(\mathbf{H}_{\mathbf{3}}\right)$ and $\left(\mathbf{H}_{\mathbf{5}}\right)$ are fulfilled, that $T(t)$ is compact for all $t>0$ and that for each $i \in \mathbb{N}_{0}$, the conditions (2.1), (2.2), (2.3) hold. Then problem (1.1)-(1.3) has at least one mild solution $u \in \mathcal{P C}(X)$.

Proof. We proceed as in the proof of Theorem 2.1. We only modify the argument to establish that the map $\Gamma_{i}: B_{R_{1}(i)}(0) \rightarrow B_{R_{1}(i)}(0)$ has a fixed point. In fact, using that $T(t)$ is compact for $t>0$, it is easy to see that $\Gamma_{i}$ is a compact map. Therefore, the assertion is a consequence of the SchauderTychonoff theorem [13, Theorem 7.1.13].

We can obtain a simpler result when the maps $\widetilde{N}_{i}$ are completely continuous.

Corollary 2.2. Assume that conditions $\left(\mathbf{H}_{\mathbf{1}}\right)-\left(\mathbf{H}_{\mathbf{4}}\right)$ are fulfilled, the maps $\widetilde{N}_{i}$, $i \in \mathbb{N}$, are completely continuous and that for each $i \in \mathbb{N}_{0}$, conditions (2.2) and (2.3) hold. Then problem (1.1)-(1.3) has at least one mild solution $u \in$ $\mathcal{P C}(X)$.

Proof. We proceed as in the proof of Theorem 2.1. In this case, we can choose $\mu_{i}=0$.

We consider now a situation frequent in applications. Next, we say that $N_{i}$ and $f$ are uniformly Hölder-continuous if there are constants $a_{i}, b_{i} \geq 0$, $\theta_{i}, \vartheta_{i} \in(0,1)$ such that

$$
\begin{gathered}
\left\|N_{i}(t)\left(v_{2}\right)-N_{i}(t)\left(v_{1}\right)\right\| \leq a_{i}\left\|v_{2}-v_{1}\right\|_{\infty}^{\theta_{i}}, t \in\left[t_{i}, s_{i}\right], v_{2}, v_{1} \in C\left(\left[t_{i}, s_{i}\right] ; X\right), \\
\|f(t, x)-f(t, y)\| \leq b_{i}\|x-y\|^{\vartheta_{i}}, t \in\left[s_{i}, t_{i+1}\right], x, y \in X .
\end{gathered}
$$

Corollary 2.3. Assume that conditions $\left(\mathbf{H}_{\mathbf{1}}\right),\left(\mathbf{H}_{\mathbf{2}}\right),\left(\mathbf{H}_{\mathbf{4}}\right)$ and $\left(\mathbf{H}_{\mathbf{5}}\right)$ are fulfilled, and the function $f(\cdot, 0)$ is locally integrable on J. Suppose that $N_{i}$, $i \in \mathbb{N}$, and $f$ are uniformly Hölder-continuous and that for each $i \in \mathbb{N}_{0}$ condition (2.1) holds. Then the problem (1.1)-(1.3) has at least one mild solution $u \in \mathcal{P C}(X)$.

Proof. We begin by pointing out that

$$
\left\|N_{i}(t)(v)\right\| \leq\left\|N_{i}(t)(v)-N_{i}(t)(0)\right\|+\left\|N_{i}(t)(0)\right\| \leq a_{i}\|v\|_{\infty}^{\theta_{i}}+\left\|N_{i}(t)(0)\right\|
$$

for $t \in\left[t_{i}, s_{i}\right]$ and $v \in C\left(\left[t_{i}, s_{i}\right] ; X\right)$, which implies that $\nu_{i, R} \leq a_{i} R^{\theta_{i}}+$ $\sup _{t_{i} \leq t \leq s_{i}}\left\|N_{i}(t)(0)\right\|$. Since

$L_{g_{i+1}} a_{i+1} R^{\theta_{i+1}}+L_{g_{i+1}} \sup _{t_{i+1} \leq t \leq s_{i+1}}\left\|N_{i+1}(t)(0)\right\|+\sup _{t_{i+1} \leq t \leq s_{i+1}}\left\|g_{i+1}(t, 0)\right\| \leq R$,

for $R$ large enough, we can select a constant $R_{2}(i+1)$ so that $(2.3)$ is verified for any $i \in \mathbb{N}_{0}$.

It remains to prove that there are constants $R_{1}(i)$ for $i \in \mathbb{N}_{0}$ for which condition (2.2) is verified. Arguing as above,

$$
\|f(t, x)\| \leq b_{i}\|x\|^{\vartheta_{i}}+\|f(t, 0)\|, t \in\left[s_{i}, t_{i+1}\right], x \in X,
$$

and we can choose $m_{f}(t)=b_{i}, h_{f}(t)=\|f(t, 0)\|$, and $\Phi_{i}(R)=R^{\vartheta_{i}}$ for $t \in$ $\left[s_{i}, t_{i+1}\right]$. Therefore, we can choose $R_{1}(i)$ sufficiently large so that condition (2.2) is verified for all $i \in \mathbb{N}_{0}$. 
The assertion is now an immediate consequence of Theorem 2.1.

In our next result we consider the following Lipschitz conditions.

$\left(\mathbf{H}_{\mathbf{6}}\right)$ There is a function $L_{f} \in L_{l o c}^{1}\left([0, \infty) ; \mathbb{R}^{+}\right)$such that $\|f(t, x)-f(t, y)\| \leq$ $L_{f}(t)\|x-y\|$ for all $x, y \in X$ and every $t \geq 0$.

$\left(\mathbf{H}_{\mathbf{7}}\right)$ There are constants $a_{i} \geq 0$ such that

$$
\left\|N_{i}(t)\left(v_{2}\right)-N_{i}(t)\left(v_{1}\right)\right\| \leq a_{i}\left\|v_{2}-v_{1}\right\|_{\infty}, t \in\left[t_{i}, s_{i}\right], v_{2}, v_{1} \in C\left(\left[t_{i}, s_{i}\right] ; X\right) .
$$

Theorem 2.2. Assume that conditions $\left(\mathbf{H}_{\mathbf{1}}\right),\left(\mathbf{H}_{\mathbf{2}}\right),\left(\mathbf{H}_{\mathbf{6}}\right)$ and $\left(\mathbf{H}_{\mathbf{7}}\right)$ are satisfied, the function $f(\cdot, 0)$ is locally integrable on $J$, and $a_{i} L_{g_{i}}<1$ for all $i \in \mathbb{N}$. Then there exists a unique mild solution $u \in \mathcal{P C}(X)$ of problem (1.1)-(1.3).

Proof. Let $G_{i}, i \in \mathbb{N}$ be the maps given by (2.4). It follows from our hypotheses that $G_{i}$ is a contraction on $C\left(\left[t_{i}, s_{i}\right] ; X\right)$. Hence, there exists a unique $v_{i} \in C\left(\left[t_{i}, s_{i}\right] ; X\right)$ such that $G_{i} v_{i}=v_{i}$. Let $\Gamma_{i}, i \in \mathbb{N}_{0}$, be the maps given by (2.5). It is not difficult to see that there exists $n_{i} \in \mathbb{N}$ such that $\Gamma_{i}^{n_{i}}$ is a contraction on $C\left(\left[s_{i}, t_{i+1}\right] ; X\right)$. Consequently, there exists a unique solution $u_{i} \in C\left(\left[s_{i}, t_{i+1}\right] ; X\right)$ such that $\Gamma_{i} u_{i}=u_{i}$, for $i \in \mathbb{N}_{0}$.

We complete the proof defining $u(\cdot)$ by $(2.7)$.

Example 2.3. In this example we consider $N_{i}(t): C\left(\left[t_{i}, s_{i}\right] ; X\right) \rightarrow X$ given by $N_{i}(t)(v)=v(t)$. We assume that conditions $\left(\mathbf{H}_{\mathbf{1}}\right)$ and $\left(\mathbf{H}_{\mathbf{2}}\right)$ hold, $L_{g_{i}}<1$ for all $i \in \mathbb{N}$ and one of the following conditions is verified.

(i) Condition $\left(\mathbf{H}_{\mathbf{3}}\right)$ holds, $T(t)$ is compact for $t>0$ and

$$
M C_{i} \liminf _{\xi \rightarrow \infty} \frac{\Phi_{i}(\xi)}{\xi} \int_{s_{i}}^{t_{i+1}} m_{f}(\tau) d \tau<1 .
$$

(ii) Condition $\left(\mathbf{H}_{\mathbf{6}}\right)$ holds and the function $f(\cdot, 0)$ is locally integrable on $J$.

Then there exists a mild solution $u \in \mathcal{P C}(X)$ of problem (1.1)(1.3).

In fact, if (i) is fulfilled, then each map $G_{i}$ is a contraction. Consequently, there exists $v_{i} \in C\left(\left[t_{i}, s_{i}\right] ; X\right)$ such that $v_{i}(t)=g_{i}\left(t, v_{i}(t)\right)$ for $i \in \mathbb{N}$. Let $R_{2}(i)=\left\|v_{i}\left(s_{i}\right)\right\|$. It follows from (2.8) that the condition (2.2) is verified for $R_{1}(i)$ sufficiently large. We complete the proof in this case as in Corollary 2.1. If (ii) is fulfilled, since $\left(\mathbf{H}_{\mathbf{7}}\right)$ holds with constants $a_{i}=1$, the assertion is an immediate consequence of Theorem 2.2.

Example 2.4. In this example we consider $N_{i}(t): C\left(\left[t_{i}, s_{i}\right] ; X\right) \rightarrow X$ given by

$$
N_{i}(t)(v)=\int_{t_{i}}^{t} p_{i}(v(s)) d s,
$$

where $p_{i}: X \rightarrow X$ is a completely continuous and not Lipschitz continuous map. We denote $\rho_{i}(R)=\sup \left\{\left\|p_{i}(x)\right\|:\|x\| \leq R\right\}$. We assume further that $T(t)$ is compact for all $t>0$, conditions $\left(\mathbf{H}_{\mathbf{1}}\right)-\left(\mathbf{H}_{\mathbf{3}}\right)$ and $(2.8)$ hold, and

$$
L_{g_{i}}\left(s_{i}-t_{i}\right) \liminf _{\xi \rightarrow \infty} \frac{\rho_{i}(\xi)}{\xi}<1, \quad \forall i \in \mathbb{N} .
$$

Then there exists a mild solution $u \in \mathcal{P C}(X)$ of problem (1.1)-(1.3). 
In fact, since

$$
\left\|G_{i}(v)(t)\right\| \leq L_{g_{i}}\left(s_{i}-t_{i}\right) \rho_{i}(R)+\left\|g_{i}(t, 0)\right\|,
$$

for any $v \in C\left(\left[t_{i}, s_{i}\right] ; X\right)$ with $\|v\|_{\infty} \leq R$, it follows from (2.9) that there is $R_{2}(i)>0$ such that $G_{i}\left(B_{R_{2}(i)}(0)\right) \subseteq B_{R_{2}(i)}(0)$. Moreover, $G_{i}$ is a compact map on $B_{R_{2}(i)}(0)$. It is sufficient to prove that $\widetilde{N}_{i}: C\left(\left[t_{i}, s_{i}\right] ; X\right) \rightarrow$ $C\left(\left[t_{i}, s_{i}\right] ; X\right)$ is completely continuous. Let $R>0$. We consider the set $W=$ $\left\{v \in C\left(\left[t_{i}, s_{i}\right] ; X\right):\|v\|_{\infty} \leq R\right\}$. It follows from the definition of $N_{i}(t)$ that $N_{i}(W)$ is equicontinuous. Moreover, using the mean value theorem, we obtain $N_{i}(t)(W) \subseteq\left(t-t_{i}\right) \overline{c o}\left\{p_{i}(v(s)): v \in W, 0 \leq s \leq t\right\} \subseteq\left(t-t_{i}\right) \overline{c o}\left(p_{i}\left(B_{R}(0)\right)\right)$, which shows that $N_{i}(t)(W)$ is relatively compact for all $t \in\left[t_{i}, s_{i}\right]$. An application of the Ascoli-Arzelá theorem allows us to affirm that $\widetilde{N}_{i}$ is completely continuous. We complete the proof proceeding as in Corollary 2.2 and Example 2.3.

The hypotheses in our previous results are general conditions to obtain the existence of solutions. There are special cases in which we can reduce considerably these hypotheses.

Example 2.5. In this example, $N_{i}(t): C\left(\left[t_{i}, s_{i}\right] ; X\right) \rightarrow X$ is given by

$$
N_{i}(t)(v)=\int_{t_{i}}^{t} v(\tau) d \tau
$$

and we assume that conditions $\left(\mathbf{H}_{\mathbf{1}}\right)-\left(\mathbf{H}_{\mathbf{2}}\right)$ hold.

(i) If in further $\left(\mathbf{H}_{\mathbf{6}}\right)$ holds and the function $f(\cdot, 0)$ is locally integrable on $J$, then there exists a unique mild solution $u \in \mathcal{P C}(X)$ of problem (1.1)(1.3).

(ii) If in further $\left(\mathbf{H}_{\mathbf{3}}\right)$ and (2.8) hold, and $T(t)$ is compact for $t>0$, then there exists a mild solution $u \in \mathcal{P C}(X)$ of problem (1.1)-(1.3).

In fact, it is not difficult to see that $G_{i}^{n_{i}}$ is a contraction for some $n_{i} \in \mathbb{N}$ large enough and each $i \in \mathbb{N}$. This implies that $G_{i}$ has a unique fixed point in $C\left(\left[t_{i}, s_{i}\right] ; X\right)$. In the case (i), we complete the proof arguing as in the proof of Theorem 2.2, while in the case (ii), we complete the proof arguing as in Example 2.3.

\subsection{On the Existence of Bounded Mild Solutions}

In this subsection, we study the existence of bounded mild solutions of problem $(1.1)-(1.3)$ on $[0, \infty)$.

Theorem 2.3. Assume that conditions $\left(\mathbf{H}_{\mathbf{1}}\right)-\left(\mathbf{H}_{\mathbf{5}}\right)$ are fulfilled, the condition (2.1) holds and there are constants $R_{1} \geq 0, R_{2} \geq\left\|x_{0}\right\|$ such that

$$
\begin{gathered}
M C_{i} R_{2}+M \sup _{s_{i} \leq t \leq t_{i+1}} \int_{s_{i}}^{t} e^{\sigma(t-\tau)}\left(\Phi_{i}\left(R_{1}\right) m_{f}(\tau)+h_{f}(\tau)\right) d \tau \leq R_{1}, \\
L_{g_{i+1}} \nu_{i+1, R_{2}}+\sup _{t_{i+1} \leq t \leq s_{i+1}}\left\|g_{i+1}(t, 0)\right\| \leq R_{2},
\end{gathered}
$$

for each $i \in \mathbb{N}_{0}$. Then the problem (1.1)-(1.3) has at least one bounded mild solution $u \in \mathcal{P C}(X)$. 
Proof. Proceeding as in the proof of Theorem 2.1, we obtain the existence of functions $u_{i}$ and $v_{i}$ such that $\left\|u_{i}\right\|_{\infty} \leq R_{1}$ and $\left\|v_{i+1}\right\|_{\infty} \leq R_{2}$ for all $i \in \mathbb{N}_{0}$. In this case, the function $u$ given by (2.7) is a mild solution of (1.1)-(1.3) and satisfies $\sup _{t \geq 0}\|u(t)\| \leq \max \left\{R_{1}, R_{2}\right\}$.

To establish our next result, for $i \in \mathbb{N}_{0}$ we introduce the notation

$$
d_{i}= \begin{cases}\frac{1}{\sigma} e^{\sigma\left(t_{i+1}-s_{i}\right)}, & \sigma>0, \\ -\frac{1}{\sigma}, & \sigma<0, \\ t_{i+1}-s_{i}, & \sigma=0 .\end{cases}
$$

Corollary 2.4. Assume that conditions $\left(\mathbf{H}_{\mathbf{1}}\right),\left(\mathbf{H}_{\mathbf{2}}\right),\left(\mathbf{H}_{\mathbf{4}}\right)$ and $\left(\mathbf{H}_{\mathbf{5}}\right)$ are fulfilled, the maps $N_{i}$ and $f$ are uniformly Hölder-continuous, and that for each $i \in \mathbb{N}_{0}$ condition (2.1) holds. If, in addition, $M \sup _{i \in \mathbb{N}_{0}} b_{i} d_{i}<1$ and

$$
\begin{gathered}
\sup _{i \in \mathbb{N}_{0}} C_{i}<\infty, \\
\sup _{i \in \mathbb{N}} \sup _{t_{i} \leq t \leq s_{i}}\left[L_{g_{i}}\left\|N_{i}(t, 0)\right\|+\left\|g_{i}(t, 0)\right\|\right]<\infty, \\
\sup _{i \in \mathbb{N}_{0}} \int_{s_{i}}^{t_{i+1}}\|f(\tau, 0)\| d \tau<\infty, \\
\sup _{i \in \mathbb{N}} L_{g_{i}} a_{i}<1,
\end{gathered}
$$

then the problem (1.1)-(1.3) has at least one bounded mild solution $u \in$ $\mathcal{P C}(X)$.

Proof. Arguing as in the proof of Corollary 2.3, and using (2.13) and (2.15), we have that

$$
\begin{aligned}
& L_{g_{i+1}} \nu_{i+1, R}+\sup _{t_{i+1} \leq t \leq s_{i+1}}\left\|g_{i+1}(t, 0)\right\| \\
& \leq L_{g_{i+1}} a_{i+1} R^{\theta_{i+1}}+\sup _{t_{i} \leq t \leq s_{i}}\left[L_{g_{i+1}}\left\|N_{i+1}(t, 0)\right\|+\left\|g_{i+1}(t, 0)\right\|\right] \\
& \leq R
\end{aligned}
$$

for $R>0$ large enough and all $i \in \mathbb{N}_{0}$. Let denote by $R_{2} \geq\left\|x_{0}\right\|$ a constant that satisfies the above condition. Proceeding in similar way, if $v \in$ $C\left(\left[s_{i}, t_{i+1}\right] ; X\right)$ with $\|v\|_{\infty} \leq R$, using (2.12), (2.14) and the fact that $M \sup _{i \in \mathbb{N}_{0}} b_{i} d_{i}<1$, we obtain that

$$
\begin{aligned}
& M C_{i} R_{2}+M \sup _{s_{i} \leq t \leq t_{i+1}} \int_{s_{i}}^{t} e^{\sigma(t-\tau)}\|f(\tau, v(\tau))\| d \tau \\
& \leq M C_{i} R_{2}+M b_{i} d_{i} R^{\vartheta_{i}}+M C_{i} \int_{s_{i}}^{t_{i+1}}\|f(\tau, 0)\| d \tau \leq R,
\end{aligned}
$$

for $R$ large enough and all $i \in \mathbb{N}_{0}$. Denoting by $R_{1}$ this constant, we can complete the proof as in Theorem 2.3. 
To complete this section, we study the existence of bounded mild solutions when the maps $f$ and $N_{i}$ satisfy Lipschitz conditions $\left(\mathbf{H}_{\mathbf{6}}\right)$ and $\left(\mathbf{H}_{\mathbf{7}}\right)$, respectively. We introduce the notations

$$
\begin{aligned}
D_{i} & =\sup _{s_{i} \leq t \leq t_{i+1}} \int_{s_{i}}^{t} e^{\sigma(t-\tau)}\|f(\tau, 0)\| d \tau \text { and } \\
E_{i} & =e^{M \int_{s_{i}}^{t_{i+1}} L_{f}(\xi) d \xi}, \text { for } i \in \mathbb{N}_{0} .
\end{aligned}
$$

Theorem 2.4. Assume conditions $\left(\mathbf{H}_{\mathbf{1}}\right),\left(\mathbf{H}_{\mathbf{2}}\right),\left(\mathbf{H}_{\mathbf{6}}\right),\left(\mathbf{H}_{\mathbf{7}}\right),(2.12)-(2.15)$ are satisfied and

$$
\sup _{i \in \mathbb{N}_{0}} \int_{s_{i}}^{t_{i+1}} L_{f}(\xi) d \xi<\infty .
$$

Then there exists a unique bounded mild solution $u \in \mathcal{P C}(X)$ of problem (1.1)-(1.3).

Proof. Let $G_{i}$ be defined by (2.4). It follows from (2.15) that $G_{i}$ is a contraction. For each $i \in \mathbb{N}$, let $v_{i} \in C\left(\left[t_{i}, s_{i}\right] ; X\right)$ be the unique solution of $G_{i} v_{i}=v_{i}$. Then, we can estimate,

$$
\begin{aligned}
\left\|v_{i}(t)\right\| & \leq\left\|g_{i}\left(t, N_{i}(t)\left(v_{i}\right)\right)-g_{i}\left(t, N_{i}(t)(0)\right)\right\|+\left\|g_{i}\left(t, N_{i}(t)(0)\right)\right\| \\
& \leq L_{g_{i}}\left\|N_{i}(t)\left(v_{i}\right)-N_{i}(t)(0)\right\|+L_{g_{i}}\left\|N_{i}(t)(0)\right\|+\left\|g_{i}(t, 0)\right\| \\
& \leq L_{g_{i}} a_{i}\left\|v_{i}\right\|_{\infty}+\sup _{i \in \mathbb{N}} \sup _{t_{i} \leq t \leq s_{i}}\left[L_{g_{i}}\left\|N_{i}(t)(0)\right\|+\left\|g_{i}(t, 0)\right\|\right],
\end{aligned}
$$

which implies that

$$
\left\|v_{i}\right\|_{\infty} \leq \frac{1}{1-\sup _{i \in \mathbb{N}} L_{g_{i}} a_{i}} \sup _{i \in \mathbb{N}} \sup _{t_{i} \leq t \leq s_{i}}\left[L_{g_{i}}\left\|N_{i}(t)(0)\right\|+\left\|g_{i}(t, 0)\right\|\right]=R_{2}^{\prime} .
$$

We denote by $R_{2}=\max \left\{\left\|x_{0}\right\|, R_{2}^{\prime}\right\}$. In addition, et $\Gamma_{i}, i \in \mathbb{N}_{0}$, be defined by (2.5). Proceeding as in the proof of Theorem 2.2 we know that there is $u_{i} \in C\left(\left[s_{i}, t_{i+1}\right] ; X\right)$ for $i \in \mathbb{N}_{0}$ be the solution of $\Gamma_{i} u_{i}=u_{i}$. It follows from (2.5) that

$$
\begin{aligned}
& \left\|u_{i}(t)\right\| \leq M e^{\sigma\left(t-s_{i}\right)} R_{2} \\
& \quad+M \int_{s_{i}}^{t} e^{\sigma(t-\tau)} L_{f}(\tau)\left\|u_{i}(\tau)\right\| d \tau+M \int_{s_{i}}^{t} e^{\sigma(t-\tau)}\|f(\tau, 0)\| d \tau
\end{aligned}
$$

which implies that

$$
\begin{aligned}
& e^{-\sigma t}\left\|u_{i}(t)\right\| \leq M e^{-\sigma s_{i}} R_{2}+M \int_{s_{i}}^{t} e^{-\sigma \tau} L_{f}(\tau)\left\|u_{i}(\tau)\right\| d \tau \\
& \quad+M \int_{s_{i}}^{t} e^{-\sigma \tau}\|f(\tau, 0)\| d \tau,
\end{aligned}
$$

and applying the Gronwall lemma, we infer that

$$
\begin{aligned}
\left\|u_{i}(t)\right\| \leq & M e^{\sigma\left(t-s_{i}\right)} R_{2}+M \int_{s_{i}}^{t} e^{\sigma(t-\tau)}\|f(\tau, 0)\| d \tau \\
& +\int_{s_{i}}^{t} e^{\sigma t}\left[M e^{-\sigma s_{i}} R_{2}+M \int_{s_{i}}^{\tau} e^{-\sigma \xi}\|f(\xi, 0)\| d \xi\right] M L_{f}(\tau) e^{\int_{\tau}^{t} M L_{f}(\xi) d \xi} d \tau \\
\leq & M C_{i} R_{2} E_{i}+M D_{i} E_{i} .
\end{aligned}
$$


Using our hypotheses, we obtain easily that

$$
\sup _{i \in \mathbb{N}_{0}} \sup _{s_{i} \leq t \leq t_{i+1}}\left\|u_{i}(t)\right\| \leq M \sup _{i \in \mathbb{N}_{0}}\left(C_{i} R_{2} E_{i}+D_{i} E_{i}\right)<\infty .
$$

Defining the mild solution $u(\cdot)$ by $(2.7)$, we obtain that $u(\cdot)$ is bounded on $[0, \infty)$.

\subsection{On the Existence of $\mathcal{S}$-Asymptotically $\omega$-Periodic Solutions}

In this subsection we study the existence of $\mathcal{S}$-asymptotically $\omega$-periodic mild solutions for (1.1)-(1.3). Concerning the theory of $\mathcal{S}$-asymptotically $\omega$ periodic we cite the papers $[17,18,28,33]$ and the recent work [32]. Next, we need to adapt the concept of $\mathcal{S}$-asymptotically $\omega$-periodic function introduced in the cited works to include piecewise continuous functions. Initially, we recall the concept of $\mathcal{S}$-asymptotically $\omega$-periodic function.

Definition 2.2. A function $u \in C_{b}([0, \infty) ; X)$ is said to be $\mathcal{S}$-asymptotically periodic if there exists $\omega>0$ such that $\lim _{t \rightarrow \infty}[u(t+\omega)-u(t)]=0$. In this case, we say that $u(\cdot)$ is a $\mathcal{S}$-asymptotically $\omega$-periodic function.

In what follows, $S A P_{\omega}(X)$ denotes the space formed by all $X$-valued $\mathcal{S}$ asymptotically $\omega$-periodic functions provided with the norm $\|\cdot\|_{C_{b}([0, \infty) ; X)}$.

Definition 2.3. We say that a function $u \in \mathcal{P C}_{b}(X)$ is $\mathcal{I} \mathcal{S}$-asymptotically periodic if there exists $\omega>0$ such that $\lim _{t \rightarrow \infty}[u(t+\omega)-u(t)]=0$. In this case, we say that $\omega$ is an asymptotic period of $u(\cdot)$ and that $u(\cdot)$ is an $\mathcal{I S}$-asymptotically $\omega$-periodic function.

We next use the notation $\operatorname{ISAP}_{\omega}(X)$ for the space formed by all $X$ valued $\mathcal{S}$-asymptotically $\omega$-periodic functions provided with the norm $\|\cdot\|_{\mathcal{P C}(X)}$. It is not difficult to see that $I S A P_{\omega}(X)$ is a Banach space,

In the remainder of this section, we always assume that there is $m \in \mathbb{N}$ such that the impulsive points $s_{i}, t_{j}$ satisfy that $t_{i}+\frac{\omega}{2^{m}}=s_{i}$ and $s_{i}+\frac{\omega}{2^{m}}=$ $t_{i+1}$ for all $i \in \mathbb{N}_{0}$.

To simplify the text, in what follows we use the following notations. We define $g:[0, \infty) \times X \rightarrow X$ as $g(t, x)=g_{i}(t, x)$ for $t \in\left[t_{i}, s_{i}\right], g_{0}(0, x)=x_{0}$, and

$$
g(t, x)=\frac{t_{i+1}-t}{t_{i+1}-s_{i}} g_{i}\left(s_{i}, x\right)+\frac{t-s_{i}}{t_{i+1}-s_{i}} g_{i+1}\left(t_{i+1}, x\right)
$$

for $t \in\left[s_{i}, t_{i+1}\right]$ and $i \in \mathbb{N}_{0}$. It is easy to see that $g$ is continuous. Let $u \in \mathcal{P C}(X)$ and $i \in \mathbb{N}$. We denote by $u_{i} \in C\left(\left[t_{i}, s_{i}\right] ; X\right)$ the function given by $u_{i}(t)=u(t)$ for $t \in\left(t_{i}, s_{i}\right]$ and $u_{i}\left(t_{i}\right)=\lim _{t \rightarrow t_{i}^{+}} u(t)$. We define $N(t)$ : $\mathcal{P C}(X) \rightarrow X$ by $N(t)(u)=N_{i}(t)\left(u_{i}\right)$ for $t \in\left[t_{i}, s_{i}\right]$, and

$$
N(t)(u)=\frac{t_{i+1}-t}{t_{i+1}-s_{i}} N\left(s_{i}\right)(u)+\frac{t-s_{i}}{t_{i+1}-s_{i}} N\left(t_{i+1}\right)(u)
$$

for $t \in\left[s_{i}, t_{i+1}\right]$ and $i \in \mathbb{N}_{0}$. Here we set $N(0)=0$.

Some of results included in the paper [17] depend heavily on the following concept. 
Definition 2.4. A continuous function $\varphi:[0, \infty) \times X \rightarrow X$ is said to be uniformly $S$-asymptotically $\omega$-periodic on bounded sets if for every bounded subset $K$ of $X$, the set $\{\varphi(t, x): t \geq 0, x \in K\}$ is bounded and $\lim _{t \rightarrow \infty}(\varphi(t, x)-$ $\varphi(t+\omega, x))=0$ uniformly for $x \in K$.

This motivates us to establish the following definition.

Definition 2.5. We say that the family of functions $\left(g_{i}\right)_{i \in \mathbb{N}}$ is uniformly $\mathcal{I S}$ asymptotically $\omega$-periodic on bounded sets if $g$ is uniformly $S$-asymptotically $\omega$-periodic on bounded sets.

We also consider the following concept.

Definition 2.6. The family $\left(N_{i}\right)_{i \in \mathbb{N}}$ is said to be $\mathcal{I S}$-asymptotically $\omega$-periodic if the set $\{N(t)(u): t \geq 0\}$ is bounded and $N(t+\omega)(u)-N(t)(u) \rightarrow 0$ as $t \rightarrow \infty$ for each $u \in I S A P_{\omega}(X)$.

In the next statement, $C=\sup _{i \in \mathbb{N}_{0}} C_{i}$. If $\sigma \leq 0$, then $C=1$ but if $\sigma>0$, then $C=e^{\sigma \omega / 2^{m}}$.

Theorem 2.5. Assume that $f$ is continuous and conditions $\left(\mathbf{H}_{\mathbf{1}}\right),\left(\mathbf{H}_{\mathbf{6}}\right),\left(\mathbf{H}_{\mathbf{7}}\right)$, (2.13) and (2.16) are fulfilled. Suppose, that $f(\cdot)$ is uniformly $\mathcal{S}$-asymptotically $\omega$-periodic on bounded sets, the family $\left(g_{i}\right)_{i \in \mathbb{N}}$ is uniformly $\mathcal{I} \mathcal{S}$-asymptotically $\omega$-periodic on bounded sets, and the family $\left(N_{i}\right)_{i \in \mathbb{N}}$ is $\mathcal{I} \mathcal{S}$-asymptotically $\omega$ periodic. If $\Theta=\sup _{i \in \mathbb{N}} L_{g_{i}}$ is finite and $\alpha=M C \sup _{i \in \mathbb{N}} L_{g_{i}} a_{i}<1$, then there exists a unique $\mathcal{I} \mathcal{S}$-asymptotically $\omega$-periodic mild solution of problem (1.1)-(1.3).

Proof. To simplify the writing of the text, in what follows we assume that $m=1$. Moreover, to avoid introducing more notations, for a function $u \in$ $\mathcal{P C}(X)$, and an interval of type $\left[s_{i}, t_{i+1}\right]$, we identify $u$ with its restriction to the interval $\left[s_{i}, t_{i+1}\right]$. Similarly, for an interval $\left(t_{i}, s_{i}\right]$, we identify $u$ with its restriction on $\left(t_{i}, s_{i}\right]$ and $u\left(t_{i}\right)=\lim _{t \rightarrow t_{i}^{+}} u(t)$.

Let $\Gamma$ be the map defined on $\mathcal{P C}^{0}(X)=\left\{u \in \mathcal{P C}(X): u(0)=x_{0}\right\}$ by $\Gamma u(t)=G_{i} u(t)$, for $t \in\left(t_{i}, s_{i}\right], i \in \mathbb{N}$, and $\Gamma u(t)=\Gamma_{i} u(t)$, for $t \in\left[s_{i}, t_{i+1}\right]$, $i \in \mathbb{N}_{0}$, where the maps $G_{i}$ are defined by (2.4) and

$$
\Gamma_{i} u(t)=T\left(t-s_{i}\right)\left(G_{i}(u)\left(s_{i}\right)\right)+\int_{s_{i}}^{t} T(t-\tau) f(\tau, u(\tau)) d \tau, \quad \text { for } t \in\left[s_{i}, t_{i+1}\right] .
$$

It is clear that $\Gamma: \mathcal{P C}^{0}(X) \rightarrow \mathcal{P C}^{0}(X)$.

We separate the rest of the proof in three steps.

First step Initially we will show that $\Gamma$ takes bounded functions into bounded functions. Let $u \in \mathcal{P C}^{0}(X)$ be a bounded function. For $t \in\left[t_{i}, s_{i}\right], i \in \mathbb{N}$, from

$$
\left\|g_{i}\left(t, N_{i}(t)(u)\right)\right\| \leq L_{g_{i}} a_{i}\|u\|_{\infty}+L_{g_{i}}\left\|N_{i}(t)(0)\right\|+\left\|g_{i}(t, 0)\right\|,
$$

the conditions on $\alpha$ and $\Theta$, and using (2.13) we obtain that $\left\{\Gamma u(t): t \in J^{\prime}\right\}$ is a bounded set. In similar way, for $t \in\left[s_{i}, t_{i+1}\right], i \in \mathbb{N}_{0}$, we have that

$$
\|\Gamma u(t)\| \leq M C\left\|u\left(s_{i}\right)\right\|+M C \int_{s_{i}}^{t} L_{f}(\tau)\|u(\tau)\| d \tau+M C \int_{s_{i}}^{t}\|f(\tau, 0)\| d \tau,
$$


and using (2.16) and the fact that $f(t, 0)$ is bounded on $[0, \infty)$, we obtain that $\{\Gamma u(t): t \in J\}$ is a bounded set. Consequently, we can consider $\Gamma$ : $\mathcal{P C}_{b}^{0}(X) \rightarrow \mathcal{P} \mathcal{C}_{b}^{0}(X)$.

Second step In this second step we will prove that $\Gamma$ is Lipschitz continuous on $\mathcal{P C}_{b}^{0}(X)$, and that there exists $n \in \mathbb{N}$ such that $\Gamma^{n}$ is a contraction. We denote $k=\sup _{i \in \mathbb{N}} L_{g_{i}} a_{i}$. Since $M C \geq 1$, we have that $0 \leq k<1$. Let $u, v \in \mathcal{P C}_{b}(X)$.

If $t \in\left[t_{i}, s_{i}\right], i \in \mathbb{N}$, then

$$
\|\Gamma v(t)-\Gamma u(t)\| \leq L_{g_{i}} a_{i} \sup _{t_{i} \leq t \leq s_{i}}\|v(t)-u(t)\| \leq k\|v-u\|_{\infty} .
$$

It is immediate that

$$
\left\|\Gamma^{n} v(t)-\Gamma^{n} u(t)\right\|_{\infty} \leq k^{n}\|v-u\|_{\infty}, \forall n \in \mathbb{N} .
$$

If $t \in\left[s_{i}, t_{i+1}\right], i \in \mathbb{N}_{0}$, then

$$
\begin{aligned}
\|\Gamma v(t)-\Gamma u(t)\| \leq & M e^{\sigma\left(t-s_{i}\right)}\left\|G_{i}(v)\left(s_{i}\right)-G_{i}(u)\left(s_{i}\right)\right\| \\
& +M \int_{s_{i}}^{t} e^{\sigma(t-\tau)} L_{f}(\tau)\|v(\tau)-u(\tau)\| d \tau \\
\leq & M C k\|v-u\|_{\infty}+M C \int_{s_{i}}^{t} L_{f}(\tau) d \tau \sup _{s_{i} \leq \tau \leq t}\|v(\tau)-u(\tau)\| .
\end{aligned}
$$

Repeating this argument, we can assert that

$$
\left\|\Gamma^{n} v(t)-\Gamma^{n} u(t)\right\| \leq\left(\alpha^{n}+\alpha^{n-1} \beta+\alpha^{n-2} \frac{\beta^{2}}{2 !}+\cdots+\frac{\beta^{n}}{n !}\right)\|v-u\|_{\infty},
$$

where we have denoted $\beta=M C \sup _{i \in \mathbb{N}_{0}} \int_{s_{i}}^{t_{i+1}} L_{f}(\tau) d \tau<\infty$. Combining these estimates, we can affirm that

$$
\left\|\Gamma^{n} v-\Gamma^{n} u\right\|_{\infty} \leq\left(\alpha^{n}+\alpha^{n-1} \beta+\alpha^{n-2} \frac{\beta^{2}}{2 !}+\cdots+\frac{\beta^{n}}{n !}\right)\|v-u\|_{\infty} .
$$

Using now (2.16) and that $\alpha<1$, we get that $\alpha^{n}+\alpha^{n-1} \beta+\alpha^{n-2} \frac{\beta^{2}}{2 !}+\cdots+$ $\frac{\beta^{n}}{n !} \rightarrow 0$, as $n \rightarrow \infty$, which implies that $\Gamma^{n}$ is a contraction for $n$ large enough.

Third step As a consequence of the Second Step, in order to establish that there exists an $\mathcal{I} \mathcal{S}$-asymptotically $\omega$-periodic mild solution it remains to show that $\Gamma\left(I S A P_{\omega}^{0}(X)\right) \subseteq I S A P_{\omega}^{0}(X)$, where $I S A P_{\omega}^{0}(X)=\left\{u \in I S A P_{\omega}(X)\right.$ : $\left.u(0)=x_{0}\right\}$.

To prove this fact, we take $u \in I S A P_{\omega}^{0}(X)$ and $t \geq 0$.

We analyze two cases. If $t \in\left[t_{i}, s_{i}\right], i \in \mathbb{N}$, then $t+\omega \in\left[t_{i+1}, s_{i+1}\right]$, and

$$
\begin{aligned}
\Gamma u(t+\omega)-\Gamma u(t)= & g(t+\omega, N(t+\omega)(u))-g(t, N(t+\omega)(u)) \\
& +g_{i}(t, N(t+\omega)(u))-g_{i}(t, N(t)(u)) .
\end{aligned}
$$

Since $\{N(\tau)(u): \tau \geq 0\}$ is a bounded set, $g(t+\omega, N(t+\omega)(u))-g(t, N(t+$ $\omega)(u)) \rightarrow 0$ as $t \rightarrow \infty$. In addition,

$$
\begin{aligned}
& \left\|g_{i}(t, N(t+\omega)(u))-g_{i}(t, N(t)(u))\right\| \leq \sup _{i \in \mathbb{N}} L_{g_{i}} \| N(t+\omega)(u) \\
& \quad-N(t)(u) \| \rightarrow 0, t \rightarrow \infty
\end{aligned}
$$


and combining these estimates,

$$
\Gamma u(t+\omega)-\Gamma u(t) \rightarrow 0, t \rightarrow \infty, t \in\left[t_{i}, s_{i}\right] .
$$

On the other hand, if $t \in\left[s_{i}, t_{i+1}\right]$, then $t+\omega \in\left[s_{i+1}, t_{i+2}\right]$. Therefore,

$$
\begin{aligned}
\Gamma u(t+\omega)-\Gamma u(t)= & T\left(t+\omega-s_{i+1}\right) G_{i+1}(u)\left(s_{i+1}\right)-T\left(t-s_{i}\right) G_{i}(u)\left(s_{i}\right) \\
& +\int_{s_{i+1}}^{t+\omega} T(t+\omega-\tau) f(\tau, u(\tau)) d \tau-\int_{s_{i}}^{t} T(t-\tau) f(\tau, u(\tau)) d \tau \\
= & T\left(t-s_{i}\right)\left(G_{i+1}(u)\left(s_{i+1}\right)-G_{i}(u)\left(s_{i}\right)\right) \\
& +\int_{s_{i}}^{t} T(t-\tau)[f(\tau+\omega, u(\tau+\omega))-f(\tau, u(\tau))] d \tau .
\end{aligned}
$$

Let $B=\{u(\tau): \tau \geq 0\}$. It follows from above that

$$
\begin{aligned}
\|\Gamma u(t+\omega)-\Gamma u(t)\| \leq & M C\left\|G_{i+1}(u)\left(s_{i+1}\right)-G_{i}(u)\left(s_{i}\right)\right\| \\
& +M \int_{s_{i}}^{t} e^{\sigma(t-\tau)}\|f(\tau+\omega, u(\tau+\omega))-f(\tau, u(\tau+\omega))\| d \tau \\
& +M \int_{s_{i}}^{t} e^{\sigma(t-\tau)}\|f(\tau, u(\tau+\omega))-f(\tau, u(\tau))\| d \tau \\
\leq & M C\left\|G_{i+1}(u)\left(s_{i+1}\right)-G_{i}(u)\left(s_{i}\right)\right\| \\
& +\frac{1}{2} \omega M C \sup _{s_{i} \leq \tau \leq t_{i+1}}\|f(\tau+\omega, x)-f(\tau, x)\| \\
& \left.+M C \int_{s_{i}}^{t} L_{f}(\tau) d \tau \sup _{s_{i} \leq \tau \leq t_{i+1}} \| u(\tau+\omega)\right)-u(\tau) \| .
\end{aligned}
$$

The first term on the right hand side of (2.18) converges to zero as $t \rightarrow \infty$ by (2.17) and using our hypotheses, we get that the other two terms also converge to zero as $t \rightarrow \infty$.

From the above steps we infer that $\Gamma^{n}$ is a contraction on $\operatorname{ISAP} P_{\omega}^{0}(X)$, which implies that there exists a unique $\mathcal{I} \mathcal{S}$-asymptotically $\omega$-periodic mild solution of (1.1)-(1.3).

In the following results, we modify some of the assumptions about functions $g_{i}$ and $N_{i}$ considered in the statement of in Theorem 2.5.

Definition 2.7. We say that the family of functions $\left(g_{i}\right)_{i \in \mathbb{N}}$ vanishes at infinite uniformly on bounded sets if for every bounded set $K \subseteq X, g(t, x) \rightarrow 0$ as $t \rightarrow \infty$ uniformly for $x \in K$.

The following result is a direct consequence of Theorem 2.5.

Theorem 2.6. Assume that $f$ is continuous and conditions $\left(\mathbf{H}_{\mathbf{1}}\right),\left(\mathbf{H}_{\mathbf{6}}\right),(2.11)$ and (2.16) are fulfilled. Suppose that $f(\cdot)$ is uniformly $\mathcal{S}$-asymptotically $\omega$ periodic on bounded sets, the family $\left(g_{i}\right)_{i \in \mathbb{N}}$ vanishes at infinite uniformly on bounded sets, and the maps $N_{i}, i \in \mathbb{N}$ are uniformly bounded on bounded sets. If the maps $\widetilde{N}_{i}: C\left(\left[t_{i}, s_{i}\right] ; X\right) \rightarrow C\left(\left[t_{i}, s_{i}\right] ; X\right), i \in \mathbb{N}$, are completely continuous, then there exists an $\mathcal{I} \mathcal{S}$-asymptotically $\omega$-periodic mild solution of (1.1)-(1.3). 
Proof. We introduce the space $Y$ consisting of all bounded continuous functions $u: J^{\prime} \rightarrow X$ provided with the norm of uniform convergence. We define $\Gamma_{2}$ on $Y$ by

$$
\Gamma_{2} u(t)=g_{i}(t, N(t)(u)), t \in\left[t_{i}, s_{i}\right], i \in \mathbb{N} .
$$

Initially we point out that as a consequence of $\left(\mathbf{H}_{\mathbf{1}}\right)$, the fact that $\widetilde{N}_{i}$ are continuous, and the family $\left(g_{i}\right)_{i \in \mathbb{N}}$ vanishes at infinite uniformly on bounded sets, it follows that $\Gamma_{2}$ is a continuous map from $Y$ into $Y$. In addition, combining $\left(\mathbf{H}_{\mathbf{1}}\right)$ with the property that $\widetilde{N}_{i}$ are completely continuous, we deduce that the maps $G_{i}$ for $i \in \mathbb{N}$ are also completely continuous. Using again that the family $\left(g_{i}\right)_{i \in \mathbb{N}}$ vanishes at infinite uniformly on bounded sets and arguing as in Lemma 1.1, we can affirm that $\Gamma_{2}$ is completely continuous. Moreover, using (2.11) we can assert that there exists a constant $R_{2}>0$ such that $\Gamma_{2}\left(B_{R_{2}}(0, Y)\right) \subseteq B_{R_{2}}(0, Y)$.

An application of the Schauder-Tychonoff theorem [13, Theorem 7.1.13] allows us to conclude the existence of a function $\bar{u} \in Y$ such that $\Gamma_{2} \bar{u}=\bar{u}$. From $\bar{u}(t)=g_{i}\left(t, N_{i}(t)(\bar{u})\right)$ for $t \in\left[t_{i}, s_{i}\right]$, we infer that $\bar{u}(t) \rightarrow 0$ as $t \in J^{\prime}$, $t \rightarrow \infty$.

We define now $\Gamma_{1}$ on $\mathcal{P} \mathcal{C}_{b}(X)$ by

$$
\Gamma_{1} u(t)= \begin{cases}T(t) x_{0}+\int_{0}^{t} T(t-\tau) f(\tau, u(\tau)) d \tau, & t \in\left[0, t_{1}\right], \\ \bar{u}(t), & t \in\left(t_{i}, s_{i}\right], \quad i \in \mathbb{N}, \\ T\left(t-s_{i}\right) \bar{u}\left(s_{i}\right)+\int_{s_{i}}^{t} T(t-\tau) f(\tau, u(\tau)) d \tau, & t \in\left(s_{i}, t_{i+1}\right], \quad i \in \mathbb{N},\end{cases}
$$

Arguing as in the proof of Theorem 2.5, we obtain that $\Gamma_{1}$ is a map from $\mathcal{P C}_{b}(X)$ into $\mathcal{P C}_{b}(X)$. Moreover, proceeding as in the third step of the proof of Theorem 2.5 we can show that $\Gamma_{1}\left(I S A P_{\omega}(X)\right) \subseteq I S A P_{\omega}(X)$. In fact, if $t \in\left[s_{i}, t_{i+1}\right]$, then $t+\omega \in\left[s_{i+1}, t_{i+2}\right]$, and.

$$
\begin{aligned}
\Gamma_{1} u(t+\omega)-\Gamma_{1} u(t)= & T\left(t+\omega-s_{i+1}\right) \bar{u}\left(s_{i+1}\right)-T\left(t-s_{i}\right) \bar{u}\left(s_{i}\right) \\
& +\int_{s_{i+1}}^{t+\omega} T(t+\omega-\tau) f(\tau, u(\tau)) d \tau \\
& -\int_{s_{i}}^{t} T(t-\tau) f(\tau, u(\tau)) d \tau \\
= & T\left(t-s_{i}\right)\left(\bar{u}\left(s_{i}+\omega\right)-\bar{u}\left(s_{i}\right)\right) \\
& +\int_{s_{i}}^{t} T(t-\tau)[f(\tau+\omega, u(\tau+\omega))-f(\tau, u(\tau))] d \tau
\end{aligned}
$$

Since $f$ is uniformly $\mathcal{S}$-asymptotically $\omega$-periodic on bounded sets and $\bar{u}\left(s_{i}\right) \rightarrow$ 0 as $i \in \mathbb{N}, i \rightarrow \infty$, then $\Gamma_{1} u(t+\omega)-\Gamma_{1} u(t) \rightarrow 0$ as $t \rightarrow \infty$.

On the other hand, proceeding as in the proof of Theorem 2.5 we can show that

$$
\left\|\Gamma_{1}^{n} v_{2}(t)-\Gamma_{1}^{n} v_{1}(t)\right\| \leq \frac{M^{n} C^{n}}{n !}\left(\int_{s_{i}}^{t_{i+1}} L_{f}(\tau) d \tau\right)^{n}\left\|v_{2}-v_{1}\right\|_{\infty}
$$

for $n \in \mathbb{N}$. Using (2.16), we infer that there exists $n \in \mathbb{N}$ such that $\Gamma_{1}^{n}$ is a contraction. 
Combining these assertions, we infer that there is $u \in I S A P_{\omega}(X)$ such that $\Gamma_{1} u=u$. This implies that $u(t)=\bar{u}(t)$ for $t \in\left(t_{i}, s_{i}\right], i \in \mathbb{N}$, and

$$
u(t)=T\left(t-s_{i}\right) u\left(s_{i}\right)+\int_{s_{i}}^{t} T(t-\tau) f(\tau, u(\tau)) d \tau
$$

for $t \in\left(s_{i}, t_{i+1}\right]$. Hence $u(\cdot)$ is an $\mathcal{I S}$-asymptotically $\omega$-periodic mild solution of (1.1)-(1.3).

The following immediate consequence of Theorem 2.6 is more appropriate for applications.

Corollary 2.5. Assume that $f$ is continuous and conditions $\left(\mathbf{H}_{\mathbf{1}}\right),\left(\mathbf{H}_{\mathbf{6}}\right)$, (2.11) and (2.16) are fulfilled. Assume further that $f(\cdot)$ is uniformly $\mathcal{S}$-asymptotically $\omega$-periodic on bounded sets, the family $\left(g_{i}\right)_{i \in \mathbb{N}}$ vanishes at infinite uniformly on bounded sets, and the maps $N_{i}, i \in \mathbb{N}$ are uniformly bounded on bounded sets. If the set $\left\{g_{i}(\cdot, x): x \in B\right\}$ is relatively compact in $C\left(\left[t_{i}, s_{i}\right] ; X\right)$ for each bounded set $B \subseteq X$, and the set $\left\{\widetilde{N}_{i}(u): u \in W\right\}$ is equicontinuous in $C\left(\left[t_{i}, s_{i}\right] ; X\right)$ for each bounded set $W \subseteq C\left(\left[t_{i}, s_{i}\right] ; X\right)$ and $i \in \mathbb{N}$, then there exists an $\mathcal{I S}$-asymptotically $\omega$-periodic mild solution of problem (1.1)-(1.3).

Proof. It only remains to prove that the map $\Gamma_{2}$ defined by (2.19) is completely continuous. Let $W \subseteq C\left(\left[t_{i}, s_{i}\right] ; X\right)$ be a bounded set. Since $\widetilde{N}_{i}(W)$ is bounded, we obtain that the set $G_{i}(W)(t) \subseteq\left\{g_{i}(t, v): v \in \widetilde{N}_{i}(W)\right\}$ is relatively compact. Furthermore, combining $\left(\mathbf{H}_{\mathbf{1}}\right)$ with the fact that $\left\{\widetilde{N}_{i}(u)\right.$ : $u \in W\}$ is equicontinuous, we get that $G_{i}(W)$ is also equicontinuous. Consequently, $G_{i}(W)$ is relatively compact in $C\left(\left[t_{i}, s_{i}\right] ; X\right)$. We complete the proof of the assertion arguing as in the proof of Corollary 1.1 and Theorem 2.6.

Example 2.6. For each $i \in \mathbb{N}$, we consider $N_{i}(t)(v)=\int_{t_{i}}^{t} v(s) d s$ and $g_{i}(t, x)=$ $\varphi_{i}(t) Q_{i}(x)$, where $\varphi_{i}:\left[t_{i}, s_{i}\right] \rightarrow \mathbb{R}$ is a continuous function such that $\varphi_{i}(t) \rightarrow$ $0, t \in\left[t_{i}, s_{i}\right], t \rightarrow \infty$, and $Q_{i}: X \rightarrow X$ is a Lipschitz continuous and completely continuous map.

We assume that conditions $\left(\mathbf{H}_{\mathbf{1}}\right),\left(\mathbf{H}_{\mathbf{6}}\right),(2.11)$ and (2.16) are fulfilled and that $f$ is continuous and uniformly $\mathcal{S}$-asymptotically $\omega$-periodic on bounded sets. Then there exists an $\mathcal{I S}$-asymptotically $\omega$-periodic mild solution of problem (1.1)-(1.3). In fact, it is not difficult to see that hypotheses of Corollary 2.5 hold.

\section{Applications}

In this section, we will study the problem of heat conduction in a metal bar subjected to impulses that are maintained during predetermined time intervals. To simplify the exposition, we shall only consider a bar located on the interval $[0, \pi]$. 
Specifically, we consider a problem described by the equations

$$
\begin{aligned}
\frac{\partial}{\partial t} w(t, \xi) & =\frac{\partial^{2}}{\partial \xi^{2}} w(t, \xi)+F(t, w(t, \xi)), \quad(t, \xi) \in \cup_{i=0}^{\infty}\left[s_{i}, t_{i+1}\right] \times[0, \pi] \\
w(t, 0) & =w(t, \pi)=0, \quad t \in[0, \infty) \\
w(0, \xi) & =z(\xi), \quad \xi \in[0, \pi] \\
w(t, \xi) & =p_{i}\left(t, q_{i}(t, w(\cdot, \xi))\right), \quad \xi \in[0, \pi], t \in\left(t_{i}, s_{i}\right], i \in \mathbb{N}
\end{aligned}
$$

where $0=t_{0}=s_{0}<t_{1}<s_{1}<\cdots<t_{n}<s_{n} \cdots$ are fixed real numbers, and $F \in C([0, \infty) \times \mathbb{R} ; \mathbb{R}) ; p_{i} \in C\left(\left[t_{i}, s_{i}\right] \times \mathbb{R} ; \mathbb{R}\right)$ and $q_{i}:\left(t_{i}, s_{i}\right] \times C\left(\left[t_{i}, s_{i}\right] ; \mathbb{R}\right) \rightarrow \mathbb{R}$ for all $i \in \mathbb{N}$. We assume that $p_{i}(t, 0)=0$ and $q_{i}(t, 0)=0$, and that $F, p_{i}, q_{i}$ are functions that satisfy appropriate conditions which will be specified later.

To model this problem in abstract form, as usual we consider the space $X=L^{2}([0, \pi])$ and define $u(t)=w(t, \cdot)$. For this reason, we take $z \in X$ and define the operator $A: D(A) \subseteq X \rightarrow X$ by $A x=x^{\prime \prime}$ on the domain $D(A):=\left\{x \in X: x^{\prime \prime} \in X, x(0)=x(\pi)=0\right\}$. It is well known that $A$ is the infinitesimal generator of a compact semigroup $(T(t))_{t \geq 0}$ on $X$ such that $\|T(t)\| \leq e^{-t}$ for all $t \geq 0$. Moreover, we define the functions $f$ : $[0, \infty) \times X \rightarrow X$ and $g_{i}:\left[t_{i}, s_{i}\right] \times X \rightarrow X$ by $f(t, x)(\xi)=F(t, x(\xi))$ and $g_{i}(t, x)(\xi)=p_{i}(t, x(\xi))$.

We study two alternatives for $q_{i}:\left(t_{i}, s_{i}\right] \times C\left(\left[t_{i}, s_{i}\right] ; \mathbb{R}\right) \rightarrow \mathbb{R}$ and $N_{i}(t):$ $C\left(\left[t_{i}, s_{i}\right] ; X\right) \rightarrow X$, for $t \in\left(t_{i}, s_{i}\right]$ and $i \in \mathbb{N}$.

(i) $q_{i}(t, w(\cdot, \xi))=w(t, \xi)$ and $N_{i}(t)(v)(\xi)=v(t)(\xi)$.

(ii) $q_{i}(t, w(\cdot, \xi))=\int_{t_{i}}^{t} w(\tau, \xi) d \tau$ and $N_{i}(t)(v)(\xi)=\int_{t_{i}}^{t} v(\tau)(\xi) d \tau$.

In these conditions the impulsive problem (3.1)-(3.4) can be modeled in the form (1.1)-(1.3). Next, we say that $w(\cdot)$ is a mild solution of (3.1)-(3.4) if $u(\cdot) \in \mathcal{P C}(X)$ is a mild solution of the associated abstract problem (1.1)(1.3).

The next result follows from Theorem 2.2, Theorem 2.4 and Theorem 2.5 .

Proposition 3.1. Assume that condition (i) holds and that the following properties are fulfilled.

(a) There exists a constant $L_{F} \geq 0$ such that $\left|F\left(t, \eta_{2}\right)-F\left(t, \eta_{1}\right)\right| \leq L_{F} \mid \eta_{2}-$ $\eta_{1} \mid$ for $t \geq 0$ and $\eta_{2}, \eta_{1} \in \mathbb{R}$.

(b) There are constants $0 \leq L_{i}<1$ such that $\left|p_{i}\left(t, \eta_{2}\right)-p_{i}\left(t, \eta_{1}\right)\right| \leq L_{i} \mid \eta_{2}-$ $\eta_{1} \mid$, for $t \in\left[t_{i}, s_{i}\right], \eta_{2}, \eta_{1} \in \mathbb{R}$ and $i \in \mathbb{N}$.

Then there exits a unique mild solution $w$ of problem (3.1)-(3.4). If the following additional conditions are verified.

(c) $\sup _{i \in \mathbb{N}} L_{i}<1 ; \sup _{i \in \mathbb{N}} \sup _{t_{i} \leq t \leq s_{i}}\left|p_{i}(t, 0)\right|<\infty ; \sup _{i \in \mathbb{N}_{0}}\left(t_{i+1}-s_{i}\right)<\infty$, and

$\sup _{i \in \mathbb{N}_{0}} \int_{s_{i}}^{t_{i+1}}|F(\tau, 0)| d \tau<\infty$,

then there exits a unique bounded mild solution $w$ of problem (3.1)-(3.4).

If, in addition to all the above conditions, $t_{i+1}-s_{i}=\omega / 2, s_{i}-t_{i}=\omega / 2$, $F(t+\omega, \eta)-F(t, \eta) \rightarrow 0, t \rightarrow \infty$, for $\eta \in \mathbb{R}$, and $p_{i+1}(t+\omega, \eta)-p_{i}(t, \eta) \rightarrow 0$, 
$t \rightarrow \infty, t \in\left[t_{i}, s_{i}\right]$ and $\eta \in \mathbb{R}$, then there exists an $\mathcal{I} \mathcal{S}$-asymptotically $\omega$ periodic mild solution of problem (3.1)-(3.4).

Proof. We only prove the last assertion. By comparing with the statement of Theorem 2.5 , it remains to show that $f(\cdot)$ is uniformly $\mathcal{S}$-asymptotically $\omega$ periodic on bounded sets, the family $\left(g_{i}\right)_{i \in \mathbb{N}}$ is uniformly $\mathcal{I} \mathcal{S}$-asymptotically $\omega$-periodic on bounded sets, and the family $\left(N_{i}\right)_{i \in \mathbb{N}}$ is $\mathcal{I} \mathcal{S}$-asymptotically $\omega$-periodic. Let $K \subseteq X$ be a bounded set and $x \in K$. Then

$$
|F(t+\omega, x(\xi))-F(t, x(\xi))| \rightarrow 0, t \rightarrow \infty, \xi \in[0, \pi] .
$$

Moreover, since $|F(t, \eta)| \leq L_{F}|\eta|+|F(t, 0)|$ the Lebesgue dominated convergence theorem implies that

$\|f(t+\omega, x)-f(t, x)\|=\left(\int_{0}^{\pi}|F(t+\omega, x(\xi))-F(t, x(\xi))|^{2} d \xi\right)^{1 / 2} \rightarrow 0, t \rightarrow \infty$,

uniformly for $x \in K$. This shows that $f(\cdot)$ is uniformly $\mathcal{S}$-asymptotically $\omega$-periodic on bounded sets. A similar argument establishes that the family $\left(g_{i}\right)_{i \in \mathbb{N}}$ is uniformly $\mathcal{I} \mathcal{S}$-asymptotically $\omega$-periodic on bounded sets. Finally, from (i) is immediate that the family $\left(N_{i}\right)_{i \in \mathbb{N}}$ is $\mathcal{I} \mathcal{S}$-asymptotically $\omega$-periodic.

On the other hand, since in this case $T(t)$ is compact for $t>0$, proceeding as in Example 2.5 we get the following consequence.

Proposition 3.2. Assume that condition (ii) and the following properties are fulfilled.

(a) There exists a positive continuous function $\rho$ such that $\int_{s_{i}}^{t_{i+1}} \rho(\tau) d \tau<1$ for all $i \in \mathbb{N}_{0}$, and $|F(t, \eta)| \leq \rho(t)|\eta|$, for $t \geq 0$ and $\eta \in \mathbb{R}$.

(b) There are constants $L_{i} \geq 0$ such that $\left|p_{i}\left(t, \eta_{2}\right)-p_{i}\left(t, \eta_{1}\right)\right| \leq L_{i}\left|\eta_{2}-\eta_{1}\right|$, for $t \in\left[t_{i}, s_{i}\right], \eta_{1}, \eta_{2} \in \mathbb{R}$ and $i \in \mathbb{N}$.

Then there exits a mild solution $w$ of problem (3.1)-(3.4).

If the following additional conditions are verified.

(c) There is $n \in \mathbb{N}$ such that $\sup _{i \in \mathbb{N}} \frac{L_{i}^{n}}{n !}<1 ; \sup _{i \in \mathbb{N}} \sup _{t_{i} \leq t \leq s_{i}}\left|p_{i}(t, 0)\right|<$ $\infty$, and $\sup _{i \in \mathbb{N}_{0}} \int_{s_{i}}^{t_{i+1}} \rho(\tau) d \tau<1$,

then there exits a bounded mild solution $w$ of problem (3.1)-(3.4).

If, in addition to all the above conditions, $t_{i+1}-s_{i}=\omega / 2, s_{i}-t_{i}=\omega / 2$, $F(t+\omega, \eta)-F(t, \eta) \rightarrow 0, t \rightarrow \infty$, for $\eta \in \mathbb{R}$, and $p_{i+1}(t+\omega, \eta)-p_{i}(t, \eta) \rightarrow 0$, $t \rightarrow \infty, t \in\left[t_{i}, s_{i}\right]$ and $\eta \in \mathbb{R}$, then there exists an $\mathcal{I} \mathcal{S}$-asymptotically $\omega$ periodic mild solution of problem (3.1)-(3.4).

Proof. To prove the first assertion, we choose $m_{f}(t)=\rho(t)$ and $\Phi_{i}(\xi)=\xi$ for $\xi \geq 0$ and $i \in \mathbb{N}_{0}$. Thus, in this case condition [2.8 holds and the assertion is a consequence of Example 2.5(ii)].

To establish the second assertion, we proceed as in the proof of Theorem 2.6. It is not difficult to see that in this case $\Gamma_{2}: Y \rightarrow Y$ and $\Gamma_{2}^{n}$ is a contraction. Hence, there exists $\bar{u} \in Y$ such that $\Gamma_{2}(\bar{u})=\bar{u}$. We complete the proof as in Theorem 2.6. 
The last assertion is proved in similar manner to what we did to establish that type of property in Proposition 3.1.

\section{References}

[1] Abbas, S., Benchohra, M., N'Guérékata, G.M.: Topics in fractional differential equations. Springer, New York (2012)

[2] Abada, N., Benchohra, M., Hammouche, H.: Existence and controllability results for nondensely defined impulsive semilinear functional differential inclusions. J. Differ. Equ. 246(10), 3834-3863 (2009)

[3] Ahmad, S., Stamov, G.T.: Almost periodic solutions of N-dimensional impulsive competitive systems. Nonlinear Anal. Real World Appl. 10(3), 18461853 (2009)

[4] Banaś, J.; Goebel, K.: Measures of Noncompactness in Banach Spaces, Lect. Notes Pure Appl. Math. 60, Marcel Dekker, New York (1980)

[5] Benchohra, M.; Henderson, J.; Ntouyas, S.: Impulsive differential equations and inclusions. Contemporary mathematics and its applications, 2. Hindawi Publishing Corporation, New York (2006)

[6] Bothe, D.: Multivalued perturbations of $m$-accretive differential inclusions. Israel J. Math. 108, 109-138 (1998)

[7] Chu, J., Nieto, J.J.: Impulsive periodic solutions of first-order singular differential equations. Bull. Lond. Math. Soc. 40(1), 143-150 (2008)

[8] Darbo, G.: Punti uniti in trasformazioni a codominio non compatto. Rend. Sem. Mat. Univ. Padova. 24, 84-92 (1955)

[9] Fan, Z., Li, G.: Existence results for semilinear differential equations with nonlocal and impulsive conditions. J. Funct. Anal. 258(5), 1709-1727 (2010)

[10] Franco, D.; Liz, E.; Nieto, J.J.; Rogovchenko, Y.V.: A contribution to the study of functional differential equations with impulses. Math. Nachr. 218:49$60(2000)$

[11] Frigon, M., O'Regan, D.: First-order impulsive initial and periodic problems with variable moments. J. Math. Anal. Appl. 233(2), 730-739 (1999)

[12] Frigon, M., O'Regan, D.: Existence results for first-order impulsive differential equations. J. Math. Anal. Appl. 193(1), 96-113 (1995)

[13] Granas, A., Dugundji, J.: Fixed point theory. Springer, New York (2003)

[14] Górniewicz, L.: Topological fixed point theory of multivalued mappings. Springer, Dordrecht (2006)

[15] He, M., Chen, F., Li, Z.: Almost periodic solution of an impulsive differential equation model of plankton allelopathy. Nonlinear Anal. Real World Appl. 11(4), 2296-2301 (2010)

[16] Heinz, H.-P.: On the behaviour of measures of noncompactness with respect to differentiation and integration of vector-valued functions. Nonlinear Anal. 7(12), 1351-1371 (1983)

[17] Henríquez, H., Pierri, M., Táboas, P.: On $\mathcal{S}$-asymptotically $\omega$-periodic functions on Banach spaces and applications. J. Math. Anal. Appl. 343(2), 1119$1130(2008)$ 
[18] Henríquez, H., Pierri, M., Táboas, P.: Existence of $\mathcal{S}$-asymptotically $\omega$-periodic solutions for abstract neutral equations. Bull. Aust. Math. Soc. 78(3), 365$382(2008)$

[19] Hernández, E., Henríquez, H., Rabello, M.: Existence of solutions for a class of impulsive partial neutral functional differential equations. J. Math. Anal. Appl. 331(2), 1135-1158 (2007)

[20] Hernández, E., O'Regan, D.: On a new class of abstract impulsive differential equations. Proc. Amer. Math. Soc. 141(5), 1641-1649 (2013)

[21] Kou, C., Zhang, S., Wu, S.: Stability analysis in terms of two measures for impulsive differential equations. J. London Math. Soc. 2(66(1)), 142-152 (2002)

[22] Lakshmikantham, V., Bainov, D.D., Simeonov, P.S.: Theory of impulsive differential equations. Series in Modern Applied Mathematics, 6. World Scientific Publishing Co.; Inc., Teaneck (1989)

[23] Li, Y., Lu, L., Zhu, X.: Existence of periodic solutions in n-species food-chain system with impulsive. Nonlinear Anal. Real World Appl. 7(3), 414-431 (2006)

[24] Liu, L., Guo, F., Wu, C., Wu, Y.: Existence theorems of global solutions for nonlinear Volterra type integral equations in Banach spaces. J. Math. Anal. Appl. 309(2), 638-649 (2005)

[25] Liu, J.H.: Nonlinear impulsive evolution equations. Dynam. Contin. Discrete Impuls. Systems. 6(1), 77-85 (1999)

[26] Luo, Z., Jing, Z.: Periodic boundary value problem for first-order impulsive functional differential equations. Comput. Math. Appl. 55(9), 2094-2107 (2008)

[27] Marle, C.-M.: Mesures et Probabilités. Hermann, Paris (1974)

[28] Nicola, S., Pierri, M.: A note on $\mathcal{S}$-asymptotically periodic functions. Nonlinear Anal. Real World Appl. 10(5), 2937-2938 (2009)

[29] Nieto, J.J., O'Regan, D.: Variational approach to impulsive differential equations. Nonlinear Anal. Real World Appl. 10(2), 680-690 (2009)

[30] Pazy, A.: Semigroups of linear operators and applications to partial differential equations. Applied Mathematical Sciences, 44. Springer, New YorkBerlin (1983)

[31] Pierri, M., O'Regan, D., Rolnik, V.: Existence of solutions for semi-linear abstract differential equations with not instantaneous impulses. Appl. Math. Comput. 219(12), 6743-6749 (2013)

[32] Pierri, M., Rolnik, V.: On pseudo S-asymptotically periodic functions. Bull. Aust. Math. Soc. 87(2), 238-254 (2013)

[33] Pierri, M.: On $S$-Asymptotically $\omega$-Periodic functions and applications. Nonlinear Anal. 75:651-661 (2012)

[34] Rogovchenko, Y.V.: Impulsive evolution systems: main results and new trends. Dynam. Contin. Discrete Impuls. Syst. 3(1), 57-88 (1997)

[35] Rogovchenko, Y.V.: Nonlinear impulse evolution systems and applications to population models. J. Math. Anal. Appl. 207(2), 300-315 (1997)

[36] Sadovskiu, B.N.: On a fixed point principle. Funct. Anal. Appl. 1(2), 74$76(1967)$

[37] Samoilenko, A.M., Perestyuk, N.A.: Impulsive differential equations. With a preface by Yu. A. Mitropol'skii and a supplement by S. I. Trofimchuk. Translated from the Russian by Y. Chapovsky. World Scientific Series on Nonlinear 
Science. Series A: Monographs and treatises, 14. World Scientific Publishing Co., Inc., River Edge (1995)

[38] Stamov, G.T.: Almost periodic solutions in impulsive competitive systems with infinite delays. Publ. Math. Debrecen. 76(1-2), 89-100 (2010)

[39] Stamov, G.T.: Almost periodic solutions of impulsive differential equations. Lecture Notes in Mathematics, 2047. Springer, New York (2012)

[40] Wang, K., Zhu, Y.: Periodic solutions, permanence and global attractivity of a delayed impulsive prey-predator system with mutual interference. Nonlinear Anal. Real World Appl. 14(2), 1044-1054 (2013)

[41] Wang, L., Yu, M., Niu, P.: Periodic solution and almost periodic solution of impulsive Lasota-Wazewska model with multiple time-varying delays. Comput. Math. Appl. 64(8), 2383-2394 (2012)

[42] Yao, M., Zhao, A., Yan, J.: Anti-periodic boundary value problems of second order impulsive differential equations. Comput. Math. Appl. 59(12), 36173629 (2010)

[43] Yu, J.S., Tang, X.H.: Global attractivity in a delay population model under impulsive perturbations . Bull. London Math. Soc. 34(3), 319-328 (2002)

[44] Zhou, W.-X.; Peng, J.: Existence of solution to a second-order boundary value problem via noncompactness measures, Discrete Dynam. Nature Soc. 786404:16, (2012)

Michelle Pierri

Departamento de Computação e Matemática

Faculdade de Filosofia

Ciências e Letras de Ribeirão Preto. Universidade de São Paulo

Ribeirão Preto, SP, CEP 14040-901, Brazil

e-mail: michellepierri@ffclrp.usp.br

Hernán R. Henríquez

Departamento de Matemática, Universidad de Santiago

USACH, Casilla 307, Correo 2, Santiago, Chile

e-mail: hernan.henriquez@usach.cl

Andréa Prokopczyk

Instituto de Biociências, Letras e Ciências Exatas

Universidade Estadual Paulista, São José do Rio Preto

SP, CEP 15054-000, Brazil.

e-mail: andreacp@ibilce.unesp.br

Received: March 9, 2015.

Accepted: July 6, 2015. 